\title{
HIGH-ORDER TIME STEPPING SCHEMES FOR SEMILINEAR SUBDIFFUSION EQUATIONS *
}

\author{
KAI WANG ${ }^{\dagger}$ AND ZHI ZHOU ${ }^{\ddagger}$
}

\begin{abstract}
The aim of this paper is to develop and analyze high-order time stepping schemes for solving semilinear subdiffusion equations. We apply the $k$-step BDF convolution quadrature to discretize the time-fractional derivative with order $\alpha \in(0,1)$, and modify the starting steps in order to achieve optimal convergence rate. This method has already been well-studied for the linear fractional evolution equations in Jin, Li and Zhou [19], while the numerical analysis for the nonlinear problem is still missing in the literature. By splitting the nonlinear potential term into an irregular linear part and a smoother nonlinear part, and using the generating function technique, we prove that the convergence order of the corrected $\mathrm{BDF} k$ scheme is $O\left(\tau^{\min (k, 1+2 \alpha-\epsilon)}\right)$, without imposing further assumption on the regularity of the solution. Numerical examples are provided to support our theoretical results.
\end{abstract}

Keywords: semilinear subdiffusion, convolution quadrature, $k$-step BDF, initial correction, error estimate.

AMS subject classifications 2010: 65M60, 65N30, 65N15, 35R11

1. Introduction. Fractional partial differential equations (PDEs) have been drawing increasing attention over the past several decades, due to their capability to describe anomalous diffusion processes, in which the mean square variance of particle displacements grow sublinearly/superlinear with the time, instead of the linear growth for a Gaussian process. Nowadays those models have been successfully employed in many practical applications, including dynamics of single-molecular protein 22 , flow in highly heterogeneous aquifer [5] and thermal diffusion in fractal domains [34, to name but a few; see [31] for an extensive list.

The aim of this paper is to study high-order time stepping schemes for solving the initial-boundary value problem for the semilinear subdiffusion equation:

$$
\left\{\begin{aligned}
\partial_{t}^{\alpha} u-\Delta u & =f(u) & & \text { in } \Omega \times(0, T), \\
u & =0 & & \text { on } \partial \Omega \times(0, T), \\
u(0) & =u_{0} & & \text { in } \Omega,
\end{aligned}\right.
$$

where $\Omega$ denotes a bounded, convex domain in $\mathbb{R}^{d}$ with smooth boundary, and $\Delta$ denotes the Laplacian on $\Omega$ with a homogenous Dirichlet boundary condition. Here $\partial_{t}^{\alpha} u$ denotes the left-sided Caputo fractional derivative of order $\alpha \in(0,1)$ with respect to $t$ and it is defined by [21, pp.91]

$$
\partial_{t}^{\alpha} u(t):=\frac{1}{\Gamma(1-\alpha)} \int_{0}^{t}(t-s)^{-\alpha} u^{\prime}(s) \mathrm{d} s, \quad \text { with } \quad \Gamma(z):=\int_{0}^{\infty} s^{z-1} e^{-s} \mathrm{~d} s .
$$

Throughout the paper, we assume that the initial data $u_{0}$ is smooth and compatible with the homogeneous Dirichlet boundary condition, and $f: \mathbb{R} \rightarrow \mathbb{R}$ is a globally smooth function, e.g., $f \in$ $C^{3}(\mathbb{R})$. Moreover, we assume that the nonlinear subdiffusion problem (1.1) has a unique global solution $u \in C([0, T] \times \bar{\Omega})$. One typical example is the time-fractional Allen-Cahn equation, i.e., $f(u)=u-u^{3}$, whose well-posedness and smoothing properties have already been investigated in [10].

High-order time stepping schemes for solving the linear time-fractional evolution problems have been intensively studied in recent years; see [17] (and the references therein) for a concise overview. Roughly speaking, there are two prominent types of schemes: piecewise polynomial interpolation (e.g., 2, 12, 26, 40]) and convolution quadrature (CQ) (e.g., [8, 16, 44, 46]). To the first group belongs

\footnotetext{
${ }^{*}$ The research of K. Wang is partially supported by a Hong Kong RGC grant (Project No. 15300817), and that of Z. Zhou by a start-up grant from the Hong Kong Polytechnic University and Hong Kong RGC grant No. 25300818.

${ }^{\dagger}$ Department of Applied Mathematics, The Hong Kong Polytechnic University, Kowloon, Hong Kong. (kai-r.wang@connect.polyu.hk)

${ }^{\ddagger}$ Department of Applied Mathematics, The Hong Kong Polytechnic University, Kowloon, Hong Kong. (zhizhou@polyu.edu.hk, zhizhou0125@gmail.com)
} 
the popular method using a piecewise linear interpolation (also known as L1 scheme). Lin and Xu [26] developed the scheme for fractional diffusion, and analyzed the stability and convergence rate; see also [40. The discretization has a local truncation error $O\left(\tau^{2-\alpha}\right)$ where $\tau$ denotes the step size in time, provided that the solution is smooth enough in time. The argument could be extended to high-order methods using piecewise polynomial interpolation 212. In the second group, CQ developed by Lubich 27,28. provided a systematic framework to construct high-order numerical schemes, and has been the foundation of many early works. Due to its particular construction, it naturally inherits the stability and accuracy of standard linear multistep methods, which greatly facilitates the analysis of resulting numerical schemes. However, for both techniques with uniform meshes, the desired convergence rates can be obtained only if data is sufficiently smooth and compatible, which is generally not valid. Otherwise, most of popular schemes can only achieve a first-order accuracy [15, 19. For the linear problem, the desired high-order convergence rates can be restored by correcting the first several time steps [16, 19, 43, even for nonsmooth problem data. See also [25, 39] for the application of L1 scheme with graded meshes, [30,32,33 for an analysis of discontinuous Galerkin method and [6, 24, 45, for studies of spectral methods.

However, there is fewer work on nonlinear subdiffusion problems. The first rigorous analysis was given in [20, where Jin et al. proposed a general framework for mathematical and numerical analysis of the nonlinear equation (1.1) with a globally Lipschitz continuous potential term $f(u)$. A time stepping scheme based on backward Euler CQ scheme or L1 method was studied and a uniform-intime convergence rate $O\left(\tau^{\alpha}\right)$ was proved. Then it was proved in [1] that the convergence rate of the backward Euler CQ scheme is $O(\tau)$ at a fixed time even for the nonsmooth data. As far as we know, there is no theoretical study on high-order schemes for the nonlinear problem (1.1) based on confirmed solution regularity. Therefore, in this paper, we aim to study high-order time stepping schemes based on CQ generated by $k$-step BDF method. This work is motivated by our preceding studies on the corrected BDF $k$ schemes for linear subdiffusion equations [16,19.

To discretize the fractional derivative, we let $0=t_{0}<t_{1}<\ldots<t_{N}=T$ be a uniform partition of the time interval $[0, T]$, with grid points $t_{n}=n \tau$ and step size $\tau=T / N$. Upon rewriting the Caputo derivative $\partial_{t}^{\alpha} u$ as a Riemann-Liouville one [21, pp. 91], we consider the following fully implicit time stepping scheme: for the given initial value $u_{0}$, find $u_{n}, n=1,2, \ldots, N$, such that

$$
\bar{\partial}_{\tau}^{\alpha}\left(u_{n}-u_{0}\right)-\Delta u_{n}=f\left(u_{n}\right)
$$

where $u_{n}, n=1,2, \cdots, N$ are the approximations to the exact solutions $u\left(t_{n}\right)$, and $\bar{\partial}_{\tau}^{\alpha} \varphi_{n}$ denotes the convolution quadrature generated by $k$-step BDF, $k=1,2, \cdots, 6$ with the definition

$$
\bar{\partial}_{\tau}^{\alpha} \varphi_{n}:=\frac{1}{\tau^{\alpha}} \sum_{i=0}^{n} \omega_{i}^{(\alpha)} \varphi_{n-i}
$$

The coefficients $\left\{\omega_{i}^{(\alpha)}\right\}_{i=0}^{\infty}$ can be computed either by the fast Fourier transform 36 37] or recursion [42] in the following series expansion

$$
\delta_{\tau}(\xi)^{\alpha}=\frac{1}{\tau^{\alpha}} \sum_{i=0}^{\infty} \omega_{i}^{(\alpha)} \xi^{i} \quad \text { with } \quad \delta_{\tau}(\xi)=\frac{1}{\tau} \sum_{i=1}^{k} \frac{1}{i}(1-\xi)^{i} .
$$

For linear subdiffusion problem, it has been shown in [19] that the scheme (1.2) is only first-order accurate in general. However, the optimal order $O\left(\tau^{k}\right)$ of the BDF $k$ scheme could be restored by correcting the first $k-1$ steps. For example, we split the source term $f$ into $f(t)=f(0)+(f(t)-f(0))$ and approximate $f(0)$ by $\bar{\partial}_{\tau} \partial_{t}^{-1} f(0)$, with a similar treatment of the initial data. This leads to a simple modification at the first step and restores the $O\left(\tau^{2}\right)$ accuracy for any fixed $t_{n}>0$ [8, 16, 29, This motivates us to decompose the nonlinear potential term $f(u)$ by

$$
f(u(t))=f\left(u_{0}\right)+f^{\prime}\left(u_{0}\right)\left(u(t)-u_{0}\right)+R\left(u(t) ; u_{0}\right) .
$$

Then the residue part, $R\left(u(t) ; u_{0}\right)=O\left(\left(u(t)-u_{0}\right)^{2}\right)$, is more regular in the time direction. As a 
result, the semilinear equation can be reformulated by

$$
\partial_{t}^{\alpha} u(t)-\left(\Delta+f^{\prime}\left(u_{0}\right) I\right) u(t)=f\left(u_{0}\right)-f^{\prime}\left(u_{0}\right) u_{0}+R\left(u(t) ; u_{0}\right),
$$

where $I$ denotes the identity operator. Therefore, by letting

$$
g_{0}=f\left(u_{0}\right)-f^{\prime}\left(u_{0}\right) u_{0} \text { and } A=\Delta+f^{\prime}\left(u_{0}\right) I,
$$

we can modify the BDF $k$ scheme (1.2) by

$$
\left\{\begin{array}{lll}
\bar{\partial}_{\tau}^{\alpha}\left(u-u_{0}\right)_{n}-A u_{n}=g_{0}+a_{n}^{(k)}\left(A u_{0}+g_{0}\right)+R\left(u_{n} ; u_{0}\right), & & 1 \leq n \leq k-1, \\
\bar{\partial}_{\tau}^{\alpha}\left(u-u_{0}\right)_{n}-A u_{n}=g_{0}+R\left(u_{n} ; u_{0}\right), & & k \leq n \leq N,
\end{array}\right.
$$

where the unknown coefficients $a_{n}^{(k)}$ were given in [19, Table 1].

TABLE 1

The coefficients $a_{n}^{(k)}$

\begin{tabular}{|c|ccccc|}
\hline BDF $k$ & $a_{1}^{(k)}$ & $a_{2}^{(k)}$ & $a_{3}^{(k)}$ & $a_{4}^{(k)}$ & $a_{5}^{(k)}$ \\
\hline$k=2$ & $\frac{1}{2}$ & & & & \\
\hline$k=3$ & $\frac{11}{12}$ & $-\frac{5}{12}$ & & & \\
\hline$k=4$ & $\frac{31}{24}$ & $-\frac{7}{6}$ & $\frac{3}{8}$ & & \\
\hline$k=5$ & $\frac{1181}{720}$ & $-\frac{177}{80}$ & $\frac{341}{240}$ & $-\frac{251}{720}$ & \\
\hline$k=6$ & $\frac{2837}{1440}$ & $-\frac{2543}{720}$ & $\frac{17}{5}$ & $-\frac{1201}{720}$ & $\frac{95}{288}$ \\
\hline
\end{tabular}

By rearranging terms, the modified $\mathrm{BDF} k$ scheme (1.8) is equivalent to

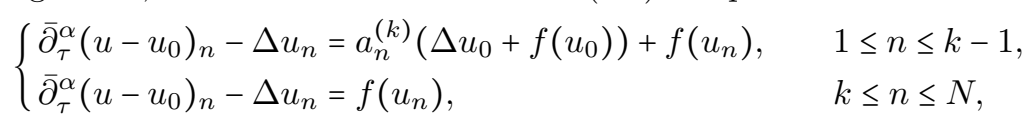

which is consistent to the BDFk scheme for the linear subdiffusion problem [16, 19].

The main result of this paper is to derive an error estimate in $L^{\infty}(\Omega)$ for the novel time stepping scheme (1.8). In particular, if $u_{0} \in\{u \in C(\bar{\Omega}), u=0$ on $\partial \Omega$, and $\Delta u \in C(\bar{\Omega})\}$, we prove that (see Theorem 3.4)

$$
\left\|u_{n}-u\left(t_{n}\right)\right\|_{L^{\infty}(\Omega)} \leq c_{T} t_{n}^{\alpha-\min (k, 1+2 \alpha-\epsilon)} \tau^{\min (k, 1+2 \alpha-\epsilon)} .
$$

This estimate is interesting, because the source term $f(u) \in W^{1+\alpha-\epsilon, 1}\left(0, T ; L^{\infty}(\Omega)\right)$ in general, which is nonsmooth in the time direction, and intuitively one only expects the convergence order $O\left(\tau^{\min (k, 1+\alpha-\epsilon)}\right)$ [19, Table 8]. However, the estimate (1.10) indicates that the best convergence rate of the BDF $k$ scheme is almost $O\left(\tau^{1+2 \alpha}\right)$. The restriction of the convergence order comes from the low regularity of the remainder $R\left(u ; u_{0}\right)$, even though the initial data $u_{0}$ is smooth and compatible with boundary condition. This phenomena contrasts sharply with its normal parabolic counterpart, i.e., $\alpha=1$. For example, in [7, it has been proved that the time stepping schemes of the semilinear parabolic equation fail to achieve the best convergence rate only if the initial data is not regular enough.

The rest of the paper is organized as follows. In section 2, we provide some preliminary results about the solution regularity which will be intensively used in error estimation. The error analysis of the time stepping scheme (1.8) is established in section 3. Then the fully discrete scheme are analyzed in section 4. Finally, in section 5, we present some numerical results which support and illustrate our theoretical findings. Throughout this paper, the notation $c$ denotes a generic constant, which may vary at different occurrences, but it is always independent of the time step size $\tau$ and spatial mesh size $h$.

2. Preliminary results. In this section, we shall present some regularity results which will be actively used in the next section. As we introduced, we always assume that the semilinear subdiffion problem (1.1) has a unique global solution $u \in C([0, T] \times \bar{\Omega})$ (e.g., the time-fractional Allen-Cahn 
equation [10]).

2.1. Solution representation. First, we introduce a representation of the solution to problem (1.1) by Laplace transform. For simplicity, we let $g(t):=f(u(t))$ and $w(t):=u(t)-u_{0}$. Then it is easy to see that the function $w(t)$ satisfies the equation

$$
\partial_{t}^{\alpha} w(t)-\Delta w(t)=\Delta u_{0}+g(t)
$$

with initial condition $w(0)=0$. Taking Laplace transform, denoted by ${ }^{\wedge}$, we have

$$
z^{\alpha} \hat{w}(z)-\Delta \hat{w}(z)=z^{-1} \Delta u_{0}+\hat{g}(z)
$$

which implies that $\hat{w}(z)=\left(z^{\alpha}-\Delta\right)^{-1}\left(z^{-1} \Delta u_{0}+\hat{g}(z)\right)$. With inverse Laplace transform and convolution rule, the solution $u(t)$ can be explicitly expressed by

$$
u(t)=(I+F(t) \Delta) u_{0}+\int_{0}^{t} E(t-s) f(u(s)) \mathrm{d} s,
$$

where the operators $F(t)$ and $E(t)$ are defined by

$$
F(t)=\frac{1}{2 \pi \mathrm{i}} \int_{\Gamma_{\theta, \delta}} e^{z t} z^{-1}\left(z^{\alpha}-\Delta\right)^{-1} \mathrm{~d} z \quad \text { and } \quad E(t)=\frac{1}{2 \pi \mathrm{i}} \int_{\Gamma_{\theta, \delta}} e^{z t}\left(z^{\alpha}-\Delta\right)^{-1} \mathrm{~d} z
$$

respectively, where $\Gamma_{\theta, \delta}$ denotes the integral contour

$$
\Gamma_{\theta, \delta}=\{z \in \mathbb{C}:|z|=\delta,|\arg z| \leq \theta\} \cup\left\{z \in \mathbb{C}: z=\rho e^{ \pm \mathrm{i} \theta}, \rho \geq \delta\right\},
$$

oriented with an increasing imaginary part with a fixed angle $\theta \in(\pi / 2, \pi)$.

In this paper, we shall derive some estimates in $L^{\infty}(\Omega)$ norm, which requires the resolvent estimate. Let us consider the second-order partial differential operator

$$
L u=-\Delta u+q u,
$$

with the homogeneous Dirichlet boundary condition. Here we assume that $q \in L^{\infty}(\Omega)$ and $q(x) \geq 0$ for all $x \in \Omega$. This implies that $L$ is positively definite, i.e.,

$$
(L u, u) \geq c\|\nabla u\|_{L^{2}(\Omega)}^{2}, \quad \text { for all } u \in H_{0}^{1}(\Omega) .
$$

Then the following resolvent estimate holds: for any angle $\phi \in(\pi / 2, \pi)$ (see [3, Theorem 1.1], 4, Theorem 2.1] or [38, Theorem 1])

$$
\left\|(z+L)^{-1}\right\|_{C(\bar{\Omega}) \rightarrow C(\bar{\Omega})} \leq c|z|^{-1} \quad \text { for } z \in \Sigma_{\phi}=\{z \in \mathbb{C} \backslash\{0\}: \arg (z) \in(-\phi, \phi)\} .
$$

From now on, we assume that the initial condition $u_{0}$ is smooth enough and compatible to the homogenous Dirichlet boundary condition, i.e.,

$$
u_{0} \in D=\{u \in C(\bar{\Omega}), u=0 \text { on } \partial \Omega \text {, and } \Delta u \in C(\bar{\Omega})\} .
$$

Then by the resolvent estimate (2.3), it is easy to observe that the operators $F$ and $E$, defined in (2.2), satisfy the following regularity estimate that for $\ell=0,1,2, \ldots$,

$$
t\left\|\partial_{t}^{(\ell)} E(t) v\right\|_{L^{\infty}(\Omega)}+\left\|\partial_{t}^{(\ell)} F(t) v\right\|_{L^{\infty}(\Omega)} \leq c t^{\alpha-\ell}\|v\|_{L^{\infty}(\Omega)} \quad \forall v \in C(\bar{\Omega}) .
$$

The estimates with $L^{2}(\Omega)$-norm have already been confirmed in [20, Lemma 3.4] by using resolvent estimate. The proof of (2.5) is similar to that, and hence is omitted here.

2.2. Solution regularity. With the help of (2.5), we are ready to state the following lemma on the regularity of the solution to the nonlinear subdiffusion equation (1.1).

Theorem 2.1. We assume that $u_{0} \in D$ with the space $D$ defined by (2.4). Besides, suppose that the problem (1.1) has a unique global solution $u \in C([0, T] \times \Omega)$. Then $u \in C^{\alpha}([0, T] ; C(\bar{\Omega})) \cap$ $C^{\ell}((0, T] ; C(\bar{\Omega})), \ell=1,2,3$, and it satisfies the a priori estimate

$$
\left\|\partial_{t}^{\ell} u(t)\right\|_{L^{\infty}(\Omega)} \leq c t^{\alpha-\ell}, \quad \text { for } \ell=1,2,3,
$$

where the constant $c$ depends on $\alpha, T$ and $u_{0}$. 
Proof. The Hölder continuity $u \in C^{\alpha}([0, T] ; C(\bar{\Omega}))$ and the estimate (2.6) with $\ell=1$ are direct results of the solution representation (2.1), the estimate (2.5), and the Banach fixed point theorem. The argument is identical to the proof of [20, Theorem 3.1], and hence omitted here.

Now we turn to the estimate (2.6) with $\ell=2$, which requires more discussion. First, we take derivative on the solution representation (2.1) and obtain

$$
\begin{aligned}
u^{\prime}(t) & =\frac{d}{d t} F(t) \Delta u_{0}+\frac{d}{d t} \int_{0}^{t} E(s) f(u(t-s)) \mathrm{d} s \\
& =E(t)\left[\Delta u_{0}+f\left(u_{0}\right)\right]+\int_{0}^{t} E(s) f^{\prime}(u(t-s)) u^{\prime}(t-s) \mathrm{d} s,
\end{aligned}
$$

where we use the fact that $F^{\prime}(t)=E(t)$. Here we note that both $E(t)$ and $u^{\prime}(t)$ are weakly singular near $t=0$. Therefore, we multiply $t^{2-\alpha}$ on (2.7) to compensate for the singularity before differentiation. Then

$$
\begin{aligned}
t^{2-\alpha} u^{\prime}(t)= & t^{2-\alpha} E(t)\left[\Delta u_{0}+f\left(u_{0}\right)\right]+t^{2-\alpha} \int_{0}^{t} E(s) f^{\prime}(u(t-s)) u^{\prime}(t-s) \mathrm{d} s \\
= & t^{2-\alpha} E(t)\left(\Delta u_{0}+f\left(u_{0}\right)\right)+t^{1-\alpha} \int_{0}^{t}(t-s) E(t-s) f^{\prime}(u(s)) u^{\prime}(s) \mathrm{d} s \\
& +t^{1-\alpha} \int_{0}^{t} E(s) f^{\prime}(u(t-s))(t-s) u^{\prime}(t-s) \mathrm{d} s \\
= & : \sum_{i=1}^{3} I_{i}(t) .
\end{aligned}
$$

After taking derivative of the first term $I_{1}$, we apply the estimate (2.5) to obtain that

$$
\begin{aligned}
\left\|\partial_{t} I_{1}(t)\right\|_{L^{\infty}(\Omega)} & =\left\|\left((2-\alpha) t^{1-\alpha} E(t)+t^{2-\alpha} E^{\prime}(t)\right)\left[\Delta u_{0}+f\left(u_{0}\right)\right]\right\|_{L^{\infty}(\Omega)} \\
& \leq c t^{1-\alpha}\left\|E(t)\left[\Delta u_{0}+f\left(u_{0}\right)\right]\right\|_{L^{\infty}(\Omega)}+t^{2-\alpha}\left\|E^{\prime}(t)\left[\Delta u_{0}+f\left(u_{0}\right)\right]\right\|_{L^{\infty}(\Omega)} \\
& \leq c\left\|\Delta u_{0}+f\left(u_{0}\right)\right\|_{L^{\infty}(\Omega)}
\end{aligned}
$$

where we use the fact that $\Delta u_{0}+f\left(u_{0}\right) \in C(\bar{\Omega})$. The derivative of the second term $I_{2}$ in (2.8) can be estimate analogously. Using the estimate (2.5), we have

$$
\lim _{t \rightarrow 0}\|t E(t)\|_{C(\bar{\Omega}) \rightarrow C(\bar{\Omega})}=0
$$

which together with the triangle's inequality and (2.6) for $\ell=1$ implies that

$$
\begin{aligned}
\left\|\partial_{t} I_{2}(t)\right\|_{L^{\infty}(\Omega)} \leq & c t^{-\alpha} \int_{0}^{t}(t-s)\left\|E(t-s) f^{\prime}(u(s)) u^{\prime}(s)\right\|_{L^{\infty}(\Omega)} \mathrm{d} s \\
& +c t^{1-\alpha} \int_{0}^{t}\left\|E(t-s) f^{\prime}(u(s)) u^{\prime}(s)\right\|_{L^{\infty}(\Omega)} \mathrm{d} s \\
& +c t^{1-\alpha} \int_{0}^{t}(t-s)\left\|E^{\prime}(t-s) f^{\prime}(u(s)) u^{\prime}(s)\right\|_{L^{\infty}(\Omega)} \mathrm{d} s \\
\leq & c t^{-\alpha} \int_{0}^{t}(t-s)^{\alpha} s^{\alpha-1} \mathrm{~d} s+c t^{1-\alpha} \int_{0}^{t}(t-s)^{\alpha-1} s^{\alpha-1} \mathrm{~d} s \leq c_{T} .
\end{aligned}
$$

Similarly, the derivative of the third term can be bounded by

$$
\begin{aligned}
&\left\|\partial_{t} I_{3}(t)\right\|_{L^{\infty}(\Omega)} \leq c t^{-\alpha} \int_{0}^{t} s\left\|E(t-s) f^{\prime}(u(s)) u^{\prime}(s)\right\|_{L^{\infty}(\Omega)} \mathrm{d} s \\
&+c t^{1-\alpha} \int_{0}^{t}\left\|E(t-s)\left[f^{\prime}(u(s)) u^{\prime}(s)+s f^{\prime \prime}(u(s))\left(u^{\prime}(s)\right)^{2}\right]\right\|_{L^{\infty}(\Omega)} \mathrm{d} s \\
&+c t^{1-\alpha} \int_{0}^{t} s\left\|E(t-s) f^{\prime}(u(s)) u^{\prime \prime}(s)\right\|_{L^{\infty}(\Omega)} \mathrm{d} s \\
& \leq c_{T}+c t^{1-\alpha} \int_{0}^{t}(t-s)^{\alpha-1} s\left\|u^{\prime \prime}(s)\right\|_{L^{\infty}(\Omega)} \mathrm{d} s . \\
& 5
\end{aligned}
$$


As a result, we achieve at

$$
\left\|\partial_{t}\left[t^{2-\alpha} u^{\prime}(t)\right]\right\|_{L^{\infty}(\Omega)} \leq c+c t^{1-\alpha} \int_{0}^{t}(t-s)^{\alpha-1} s\left\|u^{\prime \prime}(s)\right\|_{L^{\infty}(\Omega)} \mathrm{d} s .
$$

Then we apply (2.6) for $\ell=1$ again and use the triangle inequality to obtain that

$$
t^{2-\alpha}\left\|u^{\prime \prime}(t)\right\|_{L^{\infty}(\Omega)} \leq c+c t^{1-\alpha} \int_{0}^{t}(t-s)^{\alpha-1} s\left\|u^{\prime \prime}(s)\right\|_{L^{\infty}(\Omega)} \mathrm{d} s .
$$

In order to derive a uniform bound of $t^{2-\alpha}\left\|u^{\prime \prime}(t)\right\|_{L^{\infty}(\Omega)}$, we multiply $e^{-\sigma t}$ on the inequality (2.9) for some parameter $\sigma>0$ to be determined, and obtain that

$$
\begin{aligned}
& e^{-\sigma t} t^{2-\alpha}\left\|u^{\prime \prime}(t)\right\|_{L^{\infty}(\Omega)} \\
\leq & c e^{-\sigma t}+c e^{-\sigma t} \int_{0}^{t} t^{1-\alpha}(t-s)^{\alpha-1} s\left\|u^{\prime \prime}(s)\right\|_{L^{\infty}(\Omega)} \mathrm{d} s \\
\leq & c e^{-\sigma t}+c\left[\max _{t \in[0, T]} e^{-\sigma t} t^{2-\alpha}\left\|u^{\prime \prime}(t)\right\|_{L^{\infty}(\Omega)}\right] \int_{0}^{t} t^{1-\alpha}(t-s)^{\alpha-1} e^{-\sigma(t-s)} s^{\alpha-1} \mathrm{~d} s \\
\leq & c e^{-\sigma t}+c(T / \sigma)^{\frac{\alpha}{2}} \max _{t \in[0, T]} e^{-\sigma t} t^{2-\alpha}\left\|u^{\prime \prime}(t)\right\|_{L^{\infty}(\Omega)},
\end{aligned}
$$

where we use the estimate that

$$
\begin{aligned}
\int_{0}^{t} t^{1-\alpha}(t-s)^{\alpha-1} e^{-\sigma(t-s)} s^{\alpha-1} \mathrm{~d} s & =t^{\alpha} \int_{0}^{1} e^{-\sigma t s} s^{\alpha-1}(1-s)^{\alpha-1} \mathrm{~d} s \\
& =(t / \sigma)^{\frac{\alpha}{2}} \int_{0}^{1}\left[e^{-\sigma t s}(\sigma t s)^{\frac{\alpha}{2}}\right] s^{\frac{\alpha}{2}-1}(1-s)^{\alpha-1} \mathrm{~d} s \\
& \leq c(t / \sigma)^{\frac{\alpha}{2}} \int_{0}^{1} s^{\frac{\alpha}{2}-1}(1-s)^{\alpha-1} \mathrm{~d} s \leq c(T / \sigma)^{\frac{\alpha}{2}}
\end{aligned}
$$

Finally, by choosing a sufficient large $\lambda$ such that $2 c(T / \sigma)^{\frac{\alpha}{2}}<1$, we obtain that

$$
\max _{s \in[0, T]} e^{-\lambda s} s^{2-\alpha}\left\|u^{\prime \prime}(s)\right\|_{L^{\infty}(\Omega)} \leq c,
$$

which confirms the assertion (2.6) with $\ell=2$.

Now we turn to the case $\ell=3$ and give a brief proof. The basic idea of this argument is identical to that of $\ell=2$. With the definition of $I_{i}$ in (2.8) and the estimate (2.5) of the solution operator $E(t)$, we have the bound that

$$
\left\|\partial_{t t} I_{1}(t)\right\|_{L^{\infty}(\Omega)} \leq c \sum_{k=0}^{2} t^{k-\alpha}\left\|\frac{\mathrm{d}^{k}}{\mathrm{~d} t^{k}} E(t)\left[\Delta u_{0}+f\left(u_{0}\right)\right]\right\|_{L^{\infty}(\Omega)} \leq c t^{-1} .
$$

For the second term, we use the splitting

$$
I_{2}=t^{-\alpha} \int_{0}^{t}(t-s)^{2} E(t-s) f^{\prime}(u(s)) u^{\prime}(s) \mathrm{d} s+t^{-\alpha} \int_{0}^{t}(t-s) E(t-s) s f^{\prime}(u(s)) u^{\prime}(s) \mathrm{d} s,
$$

and the fact that

$$
\lim _{t \rightarrow 0}\|t E(t)\|_{C(\bar{\Omega}) \rightarrow C(\bar{\Omega})}+\left\|t u^{\prime}(t)\right\|_{L^{\infty}(\Omega)}=0,
$$

and hence derive that

$$
\begin{aligned}
&\left\|\partial_{t t} I_{2}(t)\right\|_{L^{\infty}(\Omega)} \leq c \sum_{k=0}^{2} t^{-(2-k)-\alpha} \int_{0}^{t} \sum_{m=0}^{k}(t-s)^{2+m-k}\left\|\frac{\mathrm{d}^{m}}{\mathrm{~d} t^{m}} E(t-s)\left[f^{\prime}(u(s)) u^{\prime}(s)\right]\right\|_{L^{\infty}(\Omega)} \mathrm{d} s \\
&+c \sum_{k=0}^{1} t^{-(2-k)-\alpha} \int_{0}^{t} \| \frac{\mathrm{d}^{k}}{\mathrm{~d} t^{k}}[(t-s) E(t-s)]\left[s\left(f^{\prime}(u(s)) u^{\prime}(s)\right] \|_{L^{\infty}(\Omega)} \mathrm{d} s\right. \\
&+c t^{-2-\alpha} \int_{0}^{t} \| \frac{\mathrm{d}}{\mathrm{d} t}[(t-s) E(t-s)]\left[\partial_{s}\left(s\left(f^{\prime}(u(s)) u^{\prime}(s)\right)\right] \|_{L^{\infty}(\Omega)} \mathrm{d} s .\right. \\
& 6
\end{aligned}
$$


Then the estimates (2.5) and (2.6) with $\ell=1,2$ imply that

$$
\begin{aligned}
\left\|\partial_{t t} I_{2}(t)\right\|_{L^{\infty}(\Omega)} \leq & c \sum_{k=0}^{2} t^{-(2-k)-\alpha} \int_{0}^{t}(t-s)^{\alpha-k+1} s^{\alpha-1} \mathrm{~d} s+c \sum_{k=0}^{1} t^{-(2-k)-\alpha} \int_{0}^{t}(t-s)^{\alpha-k} s^{\alpha} \mathrm{d} s \\
& +c t^{-2-\alpha} \int_{0}^{t}(t-s)^{\alpha-1}\left(s^{\alpha-1}+s^{2 \alpha-1}\right) \mathrm{d} s \leq c t^{\alpha-1} .
\end{aligned}
$$

The same argument also works for the third term $I_{3}$ in (2.8):

$$
\begin{aligned}
\left\|\partial_{t t} I_{3}(t)\right\|_{L^{\infty}(\Omega)} \leq & c \sum_{k=0}^{2} t^{-(2-k)-\alpha} \int_{0}^{t} \sum_{m=0}^{k} s^{2+m-k}\left\|E(t-s)\left[\partial_{s}^{m}\left(f^{\prime}(u(s)) u^{\prime}(s)\right)\right]\right\|_{L^{\infty}(\Omega)} \mathrm{d} s \\
& +c \sum_{k=0}^{1} t^{-(2-k)-\alpha} \int_{0}^{t} \|(t-s) E(t-s) \partial_{s}^{m}\left[s\left(f^{\prime}(u(s)) u^{\prime}(s)\right] \|_{L^{\infty}(\Omega)} \mathrm{d} s\right. \\
& +c t^{-2-\alpha} \int_{0}^{t} \| \frac{d}{d t}[(t-s) E(t-s)]\left[\partial_{s}\left(s\left(f^{\prime}(u(s)) u^{\prime}(s)\right)\right] \|_{L^{\infty}(\Omega)} \mathrm{d} s\right. \\
\leq & c t^{\alpha-1}+t^{-\alpha} \int_{0}^{t}(t-s)^{\alpha-1} s^{2}\left\|u^{(3)}(s)\right\|_{L^{\infty}(\Omega)} \mathrm{d} s .
\end{aligned}
$$

As a result, we conclude that

$$
\left\|\partial_{t t}\left[t^{2-\alpha} u^{\prime}(t)\right]\right\|_{L^{\infty}(\Omega)} \leq c t^{-1}+c t^{-\alpha} \int_{0}^{t}(t-s)^{\alpha-1} s^{2}\left\|u^{\prime \prime}(s)\right\|_{L^{\infty}(\Omega)} \mathrm{d} s .
$$

Then we apply the estimate (2.6) for $\ell=1,2$ and obtain that

$$
\left.t^{3-\alpha} \| \partial_{t}^{3} u(t)\right]\left\|_{L^{\infty}(\Omega)} \leq c+c t^{1-\alpha} \int_{0}^{t}(t-s)^{\alpha-1} s^{2}\right\| u^{\prime \prime}(s) \|_{L^{\infty}(\Omega)} \mathrm{d} s .
$$

Finally, the arguments in (2.10)-(2.12) yield the desired assertion (2.6) for $\ell=3$.

REMARK 2.1. Under the condition that $u_{0} \in D$, it is not always valid that $(I+F(t) \Delta) u_{0} \epsilon$ $C([0, T] ; D)$. This is because $\Delta(I+F(t) \Delta) u_{0}$ is compatible with the homogeneous Dirichlet boundary condition for all $t>0$, while the initial condition $\Delta u_{0} \in C(\bar{\Omega})$. As a result, $\Delta(I+F(t) \Delta) u_{0}$ is not continuous to the initial condition with $L^{\infty}(\Omega)$ norm. However, if

$$
u_{0} \in D=\{u, \Delta u \in C(\bar{\Omega}) \text { and } u=\Delta u=0 \text { on } \partial \Omega\},
$$

then we can conclude that $(I+F(t) \Delta) u_{0} \in C([0, T] ; D)$.

2.3. Regularity of remainder $R\left(u(t) ; u_{0}\right)$. Recall the expansion of the nonlinear term in (1.5). The regularity of the remainder part $R\left(u ; u_{0}\right)$ plays an important role in the error analysis. This motivates us to derive regularity results of $R\left(u ; u_{0}\right)$ in the Bochner-Sobolev spaces. For any $s \geq 0$ and $1 \leq p<\infty$, we denote by $W^{s, p}(0, T ; B)$ the space of functions $v:(0, T) \rightarrow B$, with the norm defined by interpolation, where $B$ denotes a Banach space. Equivalently, the space is equipped with the quotient norm

$$
\|v\|_{W^{s, p}(0, T ; B)}:=\inf _{\widetilde{v}}\|\widetilde{v}\|_{W^{s, p}(\mathbb{R} ; B)},
$$

where the infimum is taken over all possible extensions $\widetilde{v}$ that extend $v$ from $(0, T)$ to $\mathbb{R}$. For any $0<s<1$, the Sobolev-Slobodeckij seminorm $|\cdot|_{W^{s, p}(0, T ; B)}$ is defined by

$$
|v|_{W^{s, p}(0, T ; B)}^{p}:=\int_{0}^{T} \int_{0}^{T} \frac{\|v(t)-v(\xi)\|_{B}^{p}}{|t-\xi|^{1+p s}} \mathrm{~d} t \mathrm{~d} \xi,
$$

and the full norm $\|\cdot\|_{W^{k+s, p}(0, T ; B)}$, with $k \geq 0$ and $k \in \mathbb{N}$, is defined by

$$
\|v\|_{W^{k+s, p}(0, T ; B)}^{p}=\sum_{m=0}^{k}\left\|\partial_{t}^{m} v\right\|_{L^{p}(0, T ; B)}^{p}+\left|\partial_{t}^{k} v\right|_{W^{s, p}(0, T ; B)}^{p} .
$$


Then the regularity of $R\left(u ; u_{0}\right)$ is shown in the following theorem.

TheOREM 2.2. Suppose that the assumptions in Theorem 2.1 hold. Then the remainder part $R\left(u ; u_{0}\right)$, which is defined by (1.5), has the regularity

$$
R\left(u(t) ; u_{0}\right) \in W^{1+2 \alpha-\epsilon, 1}(0, T ; C(\bar{\Omega})) \cap C^{3}((0, T] ; C(\bar{\Omega}))
$$

for any arbitrary small $\epsilon>0$.

Proof. By the definition of $R\left(u(t) ; u_{0}\right)$ and the integral form of the remainder in Taylor's expansion, we may rewrite $R\left(u(t) ; u_{0}\right)$ as

It is easy to observe that

$$
R\left(u(x, t) ; u_{0}\right)=\int_{u_{0}(x)}^{u(x, t)}(u(x, t)-\xi) f^{\prime \prime}(\xi) \mathrm{d} \xi .
$$

$$
\begin{aligned}
\partial_{t}^{3} R\left(u(x, t) ; u_{0}\right)= & \partial_{t}^{2}\left(u^{\prime}(x, t) \int_{u_{0}(x)}^{u(x, t)} f^{\prime \prime}(\xi) \mathrm{d} \xi\right) \\
= & u^{\prime \prime \prime}(x, t) \int_{u_{0}(x)}^{u(x, t)} f^{\prime \prime}(\xi) \mathrm{d} \xi+3 u^{\prime}(x, t) u^{\prime \prime}(x, t) f^{\prime \prime}(u(x, t)) \\
& +\left(u^{\prime}(x, t)\right)^{3} f^{\prime \prime \prime}(u(x, t)) .
\end{aligned}
$$

Then using the facts that $f$ is smooth and $u \in C^{3}((0, T] ; C(\bar{\Omega}))$ by Theorem 2.1. we conclude that $R\left(u ; u_{0}\right) \in C^{3}((0, T] ; C(\bar{\Omega}))$. Therefore, it suffices to show that $R\left(u(t) ; u_{0}\right) \in W^{1+2 \alpha-\epsilon, 1}(0, T ; C(\bar{\Omega}))$. To this end, we shall confirm this claim by investigating the following two cases.

Case 1. $\alpha \in(0,1 / 2)$. Obviously, we have $R\left(u ; u_{0}\right) \in C([0, T] \times \bar{\Omega})$. Define

$$
w(x, t)=\partial_{t} R\left(u(x, t) ; u_{0}\right)=\int_{u_{0}(x)}^{u(x, t)} u^{\prime}(x, t) f^{\prime \prime}(\xi) \mathrm{d} \xi
$$

Then we observe that

$$
\|w(t)\|_{L^{\infty}(\Omega)} \leq c\left\|u(t)-u_{0}\right\|_{L^{\infty}(\Omega)}\left\|u^{\prime}(t)\right\|_{L^{\infty}(\Omega)} \max _{t \in[0, T]}\left\|f^{\prime \prime}(u(t))\right\|_{L^{\infty}(\Omega)} \leq c t^{2 \alpha-1},
$$

where the last inequality follows from the fact that $u \in C^{\alpha}([0, T] ; C(\bar{\Omega}))$ and $\left\|u^{\prime}(t)\right\|_{L^{\infty}(\Omega)} \leq c t^{\alpha-1}$, by Theorem 2.1. Then the similar argument also yields that

$$
\begin{aligned}
\left\|w^{\prime}(t)\right\|_{L^{\infty}(\Omega)} & \leq c\left(\left\|u^{\prime \prime}(t)\right\|_{L^{\infty}(\Omega)}\left\|u(t)-u_{0}\right\|_{L^{\infty}(\Omega)}+\left\|u^{\prime}(t)\right\|_{L^{\infty}(\Omega)}^{2}\right) \max _{t \in[0, T]}\left\|f^{\prime \prime}(u(t))\right\|_{L^{\infty}(\Omega)} \\
& \leq c t^{2 \alpha-2} .
\end{aligned}
$$

Then according to the Sobolev-Slobodeckij seminorm (2.13), we have for any $\epsilon \in(0,2 \alpha)$

$$
\begin{aligned}
|w|_{W^{2 \alpha-\epsilon, 1}\left(0, T ; L^{\infty}(\Omega)\right)} & =\int_{0}^{T} \int_{0}^{T} \frac{\|w(t)-w(s)\|_{L^{\infty}(\Omega)}}{|t-s|^{2 \alpha+1-\epsilon}} \mathrm{d} t \mathrm{~d} s=\int_{0}^{T} \int_{0}^{T} \frac{\left\|\int_{s}^{t} w^{\prime}(y) \mathrm{d} y\right\|_{L^{\infty}(\Omega)}}{|t-s|^{2 \alpha+1-\epsilon}} \mathrm{d} t \mathrm{~d} s \\
& \leq \int_{0}^{T} \int_{0}^{T} \frac{\left|\int_{s}^{t}\left\|w^{\prime}(y)\right\|_{L^{\infty}(\Omega)} \mathrm{d} y\right|}{|t-s|^{2 \alpha+1-\epsilon}} \mathrm{d} t \mathrm{~d} s .
\end{aligned}
$$

Now by applying the estimate (2.14), we arrive at

$$
\begin{aligned}
|w|_{W^{2 \alpha-\epsilon, 1}\left(0, T ; L^{\infty}(\Omega)\right)} & \leq c \int_{0}^{T} \int_{0}^{T} \frac{\left|\int_{s}^{t} y^{2 \alpha-2} \mathrm{~d} y\right|}{|t-s|^{2 \alpha+1-\epsilon}} \mathrm{d} t \mathrm{~d} s=c \int_{0}^{1} \int_{0}^{1} \frac{\left|\xi^{2 \alpha-1}-\zeta^{2 \alpha-1}\right|}{|\xi-\zeta|^{2 \alpha+1-\epsilon}} \mathrm{d} \xi \mathrm{d} \zeta \\
& =c\left(\int_{0}^{1} \int_{0}^{\xi} \frac{\zeta^{2 \alpha-1}-\xi^{2 \alpha-1}}{(\xi-\zeta)^{2 \alpha+1-\epsilon}} \mathrm{d} \zeta \mathrm{d} \xi+\int_{0}^{1} \int_{\xi}^{1} \frac{\xi^{2 \alpha-1}-\zeta^{2 \alpha-1}}{(\zeta-\xi)^{2 \alpha+1-\epsilon}} \mathrm{d} \zeta \mathrm{d} \xi\right) \\
& =2 c \int_{0}^{1} \int_{0}^{\xi} \frac{\zeta^{2 \alpha-1}-\xi^{2 \alpha-1}}{(\xi-\zeta)^{2 \alpha+1-\epsilon}} \mathrm{d} \zeta \mathrm{d} \xi=2 c \int_{0}^{1} \xi^{-1+\epsilon} \mathrm{d} \xi \int_{0}^{1} \frac{t^{2 \alpha-1}-1}{(1-t)^{2 \alpha+1-\epsilon}} \mathrm{d} t \\
& \leq c_{\epsilon} \int_{0}^{1} \frac{t^{2 \alpha-1}-1}{(1-t)^{2 \alpha+1-\epsilon}} \mathrm{d} t .
\end{aligned}
$$


Then the assertion that $w \in W^{1+2 \alpha-\epsilon, 1}\left(0, T ; L^{\infty}(\Omega)\right)$ follows from the observation that

$$
\begin{aligned}
\int_{0}^{1} \frac{t^{2 \alpha-1}-1}{(1-t)^{2 \alpha+1-\epsilon}} \mathrm{d} t & \leq\left(\int_{0}^{\frac{1}{2}}+\int_{\frac{1}{2}}^{1}\right) \frac{t^{2 \alpha-1}-1}{(1-t)^{2 \alpha+1-\epsilon}} \mathrm{d} t \\
& \leq c+c \lim _{t \rightarrow 1} \frac{t^{2 \alpha-1}-1}{(1-t)^{2 \alpha-\epsilon}}+c \int_{\frac{1}{2}}^{1} \frac{t^{2 \alpha-2}}{(1-t)^{2 \alpha-\epsilon}} \mathrm{d} t \leq c .
\end{aligned}
$$

Case 2. $\alpha \in(1 / 2,1)$. In this case, using the estimate (2.14), it is easy to see that $\left\|\partial_{t} w(t)\right\|_{L^{\infty}(\Omega)} \epsilon$ $L^{1}(0, T)$. Then our aim is to show that

$$
\partial_{t t} w \in W^{2 \alpha-\epsilon-1,1}\left(0, T ; L^{\infty}(\Omega)\right)
$$

Using the expression for $\partial_{t t} w$ that

$$
\partial_{t t} w(x, t)=\left(u^{\prime}(x, t)\right)^{3} f^{\prime \prime \prime}(u(x, t))+2 u^{\prime}(x, t) u^{\prime \prime}(x, t) f^{\prime \prime}(u(x, t))+u^{\prime \prime \prime}(x, t) \int_{u_{0}(x)}^{u(x, t)} f^{\prime \prime}(\xi) \mathrm{d} \xi,
$$

and Theorem 2.1 we derive that

$$
\begin{aligned}
\left\|w_{t t}\right\|_{L^{\infty}(\Omega)} \leq & c\left\|\left(u^{\prime}(t)\right)\right\|_{L^{\infty}(\Omega)}^{3}+c\left\|u^{\prime}(t)\right\|_{L^{\infty}(\Omega)}\left\|u^{\prime \prime}(t)\right\|_{L^{\infty}(\Omega)} \\
& +c\left\|u^{\prime \prime \prime}(t)\right\|_{L^{\infty}(\Omega)}\left\|u(t)-u_{0}\right\|_{L^{\infty}(\Omega)} \\
\leq & c\left(t^{3 \alpha-3}+t^{2 \alpha-3}\right) \leq c t^{2 \alpha-3} .
\end{aligned}
$$

Recalling the Sobolev-Slobodeckij seminorm (2.13), we have for any $\epsilon \in(0,2 \alpha-1)$

$$
\begin{aligned}
\left\|w_{t}\right\|_{W^{2 \alpha-1-\epsilon, 1}\left(0, T ; L^{\infty}(\Omega)\right)} & =\int_{0}^{T} \int_{0}^{T} \frac{\left\|w_{t}(t)-w_{s}(s)\right\|_{L^{\infty}(\Omega)}}{|t-s|^{2 \alpha-\epsilon}} \mathrm{d} t \mathrm{~d} s \\
& =\int_{0}^{T} \int_{0}^{T} \frac{\left|\int_{s}^{t}\left\|\partial_{y y} w(y)\right\|_{L^{\infty}(\Omega)} \mathrm{d} y\right|}{|t-s|^{2 \alpha-\epsilon}} \mathrm{d} t \mathrm{~d} s .
\end{aligned}
$$

Then we apply the estimate (2.15) and derive that

$$
\begin{aligned}
\left\|w_{t}\right\|_{W^{2 \alpha-1-\epsilon, 1}\left(0, T ; L^{\infty}(\Omega)\right)} & \leq c \int_{0}^{T} \int_{0}^{T} \frac{\left|\int_{s}^{t} y^{2 \alpha-3} \mathrm{~d} y\right|}{|t-s|^{2 \alpha-\epsilon}} \mathrm{d} t \mathrm{~d} s=c \int_{0}^{1} \int_{0}^{1} \frac{\left|\xi^{2 \alpha-2}-\zeta^{2 \alpha-2}\right|}{|\xi-\zeta|^{2 \alpha-\epsilon}} \mathrm{d} \xi \mathrm{d} \zeta \\
& =c\left(\int_{0}^{1} \int_{0}^{\xi} \frac{\zeta^{2 \alpha-2}-\xi^{2 \alpha-2}}{(\xi-\zeta)^{2 \alpha-\epsilon}} \mathrm{d} \zeta \mathrm{d} \xi+\int_{0}^{1} \int_{\xi}^{1} \frac{\xi^{2 \alpha-2}-\zeta^{2 \alpha-2}}{(\zeta-\xi)^{2 \alpha-\epsilon}} \mathrm{d} \zeta \mathrm{d} \xi\right) \\
& =2 c \int_{0}^{1} \int_{0}^{\xi} \frac{\zeta^{2 \alpha-2}-\xi^{2 \alpha-2}}{(\xi-\zeta)^{2 \alpha-\epsilon}} \mathrm{d} \zeta \mathrm{d} \xi=2 c \int_{0}^{1} \xi^{-1+\epsilon} \mathrm{d} \xi \int_{0}^{1} \frac{t^{2 \alpha-2}-1}{(1-t)^{2 \alpha-\epsilon}} \mathrm{d} t \\
& \leq c,
\end{aligned}
$$

where the last inequality is a direct consequence of the fact that

$$
\begin{aligned}
\int_{0}^{1} \frac{t^{2 \alpha-2}-1}{(1-t)^{2 \alpha-\epsilon}} \mathrm{d} t & \leq\left(\int_{0}^{\frac{1}{2}}+\int_{\frac{1}{2}}^{1}\right) \frac{t^{2 \alpha-2}-1}{(1-t)^{2 \alpha-\epsilon}} \mathrm{d} t \\
& \leq c+c \lim _{t \rightarrow 1} \frac{t^{2 \alpha-2}-1}{(1-t)^{2 \alpha+1-\epsilon}}+c \int_{\frac{1}{2}}^{1} \frac{t^{2 \alpha-3}}{(1-t)^{2 \alpha+1-\epsilon}} \mathrm{d} t \leq c .
\end{aligned}
$$

Therefore, we obtain that $\partial_{t t} w \in W^{2 \alpha-\epsilon-1,1}\left(0, T ; L^{\infty}(\Omega)\right)$, and thus $u \in W^{2 \alpha+1-\epsilon, 1}\left(0, T ; L^{\infty}(\Omega)\right)$ for any $\alpha \in(1 / 2,1)$.

In conclusion, Case 1 and 2 together confirm the desired assertion for $\alpha \in(0,1 / 2) \cup(1 / 2,1)$. The critical case $\alpha=1 / 2$ follows directly from the result of Case 1 and hence the proof is completed.

3. Error analysis of modified BDF schemes. The aim of this section is to present a complete error analysis for the high-order time stepping scheme (1.8). To begin with, we assume that the nonlinear term is globally Lipschitz continuous, i.e., there exists a constant $c_{L}$ such that

$$
|f(s)-f(t)| \leq c_{L}|t-s| \quad \text { for all } t, s \in \mathbb{R} .
$$


We shall establish numerical analysis under the assumption (3.1), and then extend the argument to the case without that assumption.

3.1. Existence and uniqueness of the time stepping solution. In the analysis stated in the next subsection, we will always assume that the fully implicit scheme (1.8) admits a unique solution. It is easy to confirm this assumption, provided that (3.1) is valid.

In each time level, the fully implicit scheme (1.8) requires to solve a nonlinear elliptic problem

$$
\left(b_{0} I+\tau^{\alpha} \Delta\right) v=w+\tau^{\alpha} f(v)
$$

with homogeneous Dirichlet boundary condition and some function $w \in C(\bar{\Omega})$. Next we show that there exists a unique solution to (3.2) in $C(\bar{\Omega})$. By defining the operator $M: C(\bar{\Omega}) \rightarrow C(\bar{\Omega})$ as

$$
M v=\left(b_{0} I+\tau^{\alpha} \Delta\right)^{-1}\left(w+\tau^{\alpha} f(v)\right)
$$

and applying the resolvent estimate (2.3), we observe that for any $v_{1}, v_{2} \in C(\bar{\Omega})$

$$
\left\|M v_{1}-M v_{2}\right\|_{L^{\infty}(\Omega)}=\left\|\left(b_{0} \tau^{-\alpha} I+\Delta\right)^{-1}\left(f\left(v_{1}\right)-f\left(v_{2}\right)\right)\right\|_{L^{\infty}(\Omega)} \leq c c_{L} \tau^{\alpha}\left\|v_{1}-v_{2}\right\|_{L^{\infty}(\Omega)} .
$$

Then for $\tau$ small enough, $M$ is a contraction mapping, and hence there exists a unique $v \in C(\bar{\Omega})$ such that $M(v)=v$, i.e., the nonlinear elliptic problem (3.2) has a unique solution. As a result, we conclude that the fully implicit time stepping scheme (1.8) admits a unique sequence of functions $\left\{u_{n}\right\}_{n=1}^{N}$ via mathematical induction.

3.2. Error analysis of the BDF scheme for linear problem. The fundamental idea of error estimation is to apply the representation of the time stepping solution by contour integral in $\mathbb{C}$, which has been extensively used in existing studies [15, 18, 19,29, 43. We shall apply this technique to derive error estimates in $L^{\infty}(\Omega)$ norm. Note that the operator $A$ defined in (1.7) is self-adjoint, but not negative definite. However, the spectrum of $A$ has an upper bound, since $f\left(u_{0}\right) \in L^{\infty}(\Omega)$. Now we define

$$
\lambda=\max \left(1,\left\|f\left(u_{0}\right)\right\|_{L^{\infty}(\Omega)}\right),
$$

and observe that $L=\Delta+\left(f\left(u_{0}\right)-\lambda\right) I$ is self-adjoint and negative definite. According to the resolvent estimate (2.3) for $L$, we have the new resolvent estimate, for $v \in C(\bar{\Omega})$

$$
\left\|(z-A)^{-1} v\right\|_{L^{\infty}(\Omega)}=\left\|\left(z-\lambda-\left(\Delta+\left(f\left(u_{0}\right)-\lambda\right) I\right) v\right)^{-1}\right\|_{L^{\infty}(\Omega)} \leq c_{\phi}|z-\lambda|^{-1}\|v\|_{L^{\infty}(\Omega)},
$$

$$
z \in \Sigma_{\lambda, \phi}:=\{z \in \mathbb{C} \backslash\{\lambda\}:|\arg (z-\lambda)|<\phi\} \text { and } \phi \in(\pi / 2, \pi) .
$$

To analyze the fully implicit BDF scheme, we shall start with the linear problem with a timeindependent source term

$$
\partial_{t}^{\alpha} v(t)-A v(t)=g_{0} \quad \text { with } t \in(0, T], \quad \text { and } \quad v(0)=u_{0} .
$$

Then the time stepping scheme reads

$$
\left\{\begin{array}{lr}
\bar{\partial}_{\tau}^{\alpha}\left(v-u_{0}\right)_{n}-A v_{n}=g_{0}+a_{n}^{(k)}\left(A u_{0}+g_{0}\right), & 1 \leq n \leq k-1, \\
\bar{\partial}_{\tau}^{\alpha}\left(v-u_{0}\right)_{n}-A v_{n}=g_{0}, & k \leq n \leq N
\end{array}\right.
$$

with $v_{0}=u_{0}$. The next lemma gives an estimate of the difference between $v\left(t_{n}\right)$ and $v_{n}$.

LEMma 3.1. Let $v(t)$ and $v_{n}$ be the solutions of (3.6) and (3.7), respectively. We assume that the conditions in Theorem 2.1 hold true. Then there exists $\tau_{0}>0$, such that for $\tau \leq \tau_{0}$ the following error estimate holds

$$
\left\|v_{n}-v\left(t_{n}\right)\right\|_{L^{\infty}(\Omega)} \leq c \tau^{k} t_{n}^{\alpha-k}\left\|g_{0}+A u_{0}\right\|_{L^{\infty}(\Omega)}
$$

where the constant $c$ depends on $\alpha, k$ and $T$.

Proof. Let $w(t)=v(t)-u_{0}$. Then the linear problem (3.6) can be reformulated as

$$
\partial_{t}^{\alpha} w-A w=A u_{0}+g_{0} \quad \text { with } t \in(0, T], \quad \text { and } w(0)=0 .
$$


After taking Laplace transform, we derive that

$$
\widehat{w}(z)=z^{-1}\left(z^{\alpha}-A\right)^{-1}\left(A u_{0}+g_{0}\right)
$$

for any $z$ in the resolvent set of $A$. With inverse Laplace transform, the function $w(t)$ can be expressed explicitly by

$$
w(t)=\frac{1}{2 \pi \mathrm{i}} \int_{\sigma_{0}-\mathrm{i} \infty}^{\sigma_{0}+\mathrm{i} \infty} e^{z t} K(z)\left(A u_{0}+g_{0}\right) \mathrm{d} z
$$

with $\sigma_{0}$ such that $\left(\sigma_{0}\right)^{\alpha}>\lambda$, where $\lambda$ is given in (3.3) and the kernel $K(z)$ is defined by

$$
K(z)=z^{-1}\left(z^{\alpha}-A\right)^{-1} \text {. }
$$

Now we deform the integral contour and obtain that

$$
w(t)=\frac{1}{2 \pi \mathrm{i}} \int_{\Gamma_{\theta, \sigma}} e^{z t} K(z)\left(A u_{0}+g_{0}\right) \mathrm{d} z
$$

where $\sigma \in\left(\lambda^{1 / \alpha}, \sigma_{0}\right]$ and the contour $\Gamma_{\theta, \sigma}$ is defined by

$$
\Gamma_{\theta, \sigma}=\left\{z \in \mathbb{C}: z=\sigma+\rho e^{ \pm \mathrm{i} \theta}, \rho \geq 0\right\} \quad \text { with any } \theta \in(\pi / 2, \pi),
$$

oriented with an increasing imaginary part.

Similarly, we may derive the integral representation of $v_{n}$ in the complex domain. By letting $w_{n}=v_{n}-u_{0}$, we can reformulate the time stepping scheme as

$$
\left\{\begin{array}{lrl}
\bar{\partial}_{\tau}^{\alpha} w_{n}-A w_{n}=\left(1+a_{n}^{(k)}\right)\left(A u_{0}+g_{0}\right), & 1 \leq n \leq k-1, \\
\bar{\partial}_{\tau}^{\alpha} w_{n}-A w_{n}=A u_{0}+g_{0}, & k \leq n \leq N
\end{array}\right.
$$

with $w_{0}=0$. By multiplying $\xi^{n}$ on (3.11) and taking summation over $n$, we have

$$
\sum_{n=1}^{\infty} \xi^{n} \bar{\partial}_{\tau}^{\alpha} w_{n}-\sum_{n=1}^{\infty} \xi^{n} A w_{n}=\left(\sum_{n=1}^{\infty} \xi^{n}+\sum_{n=1}^{k-1} \xi^{n} a_{n}^{(k)}\right)\left(A u_{0}+g_{0}\right)
$$

For any given sequence $\left(f^{n}\right)_{n=0}^{\infty}$, let $\widetilde{f}(\xi):=\sum_{n=0}^{\infty} f^{n} \xi^{n}$ denote its generating function. Since $w_{0}=0$, according to properties of discrete convolution, we have the identity

$$
\sum_{n=1}^{\infty} \xi^{n} \bar{\partial}_{\tau}^{\alpha} w_{n}=\delta_{\tau}(\xi)^{\alpha} \widetilde{W}(\xi)
$$

where $\delta_{\tau}(\xi)$ denotes the generating function of the standard BDF $k$ method (1.4). Therefore

$$
\left(\delta_{\tau}(\xi)^{\alpha}-A\right) \widetilde{w}=\left(\frac{\xi}{1-\xi}+\sum_{n=1}^{k-1} \xi^{n} a_{n}^{(k)}\right)\left(A u_{0}+g_{0}\right) .
$$

By the $A\left(\theta_{k}\right)$-stability of the BDFk method [13, pp. 251], for any $\xi$ such that $|\xi|=\rho \in\left(0, \frac{1}{2}\right]$, there exists $\tau_{0}$ small enough such that $\delta_{\tau_{0}}\left(\frac{1}{2}\right)^{\alpha}>\lambda+c_{0}$, and we can find an angle $\theta_{0} \in(\pi / 2, \pi)$ such that $\delta_{\tau}(\xi)^{\alpha} \in \Sigma_{\lambda+c_{0}, \theta_{0}}$ for all $\tau \leq \tau_{0}$ and hence the operator $\left(\delta_{\tau}(\xi)^{\alpha}-A\right)$ is invertible. Then

$$
\widetilde{w}(\xi)=K\left(\delta_{\tau}(\xi)\right) \tau^{-1} \mu(\xi)\left(A u_{0}+g_{0}\right),
$$

where $\mu(\xi)=\delta(\xi)\left(\frac{\xi}{1-\xi}+\sum_{n=1}^{k-1} \xi^{n} a_{n}^{(k)}\right)$.

Let $\rho \in\left(0, \frac{1}{2}\right]$ and $\tau \leq \tau_{0}$, it is easy to see that $\widetilde{w}(\xi)$ is analytic with respect to $\xi$ in the circle $|\xi|=\rho$ on the complex plane, then with the change of variables $\xi=e^{-z \tau}$ and Cauchy's integral formula, we have the following expression

$$
\begin{aligned}
w_{n} & =\frac{1}{2 \pi \mathrm{i}} \int_{|\xi|=\rho} \xi^{-n-1} \widetilde{w}(\xi) \mathrm{d} \xi \\
& =\frac{1}{2 \pi \mathrm{i}} \int_{\Gamma^{\tau}} e^{z t_{n}} K\left(\delta_{\tau}\left(e^{-z \tau}\right)\right) \mu\left(e^{-z \tau}\right)\left(A u_{0}+g_{0}\right) \mathrm{d} z \\
& =\frac{1}{2 \pi \mathrm{i}} \int_{\Gamma_{\theta, \sigma_{0}}^{\tau}} e^{z t_{n}} K\left(\delta_{\tau}\left(e^{-z \tau}\right)\right) \mu\left(e^{-z \tau}\right)\left(A u_{0}+g_{0}\right) \mathrm{d} z
\end{aligned}
$$


where $\Gamma^{\tau}:=\{z=-\ln (\rho) / \tau+\mathrm{i} y: y \in \mathbb{R},|y| \leq \pi / \tau\}$ and $\Gamma_{\theta, \sigma}^{\tau}=\left\{z \in \Gamma_{\theta, \sigma}:|\operatorname{Im}(z)| \leq \pi / \tau\right\}$ with $\sigma=$ $-\ln \left(\frac{1}{2}\right) / \tau_{0}$. The deformation of contour from $\Gamma^{\tau}$ to $\Gamma_{\theta, \sigma_{0}}^{\tau}$ in the last equation is achieved due to the analyticity and periodicity of the function $e^{z t_{n}} K\left(\delta_{\tau}\left(e^{-z \tau}\right)\right) \mu\left(e^{-z \tau}\right)$. Then there exists $\theta \in(\pi / 2, \pi)$ close to $\pi / 2$ such that $\delta_{\tau}\left(e^{-z \tau}\right)^{\alpha} \in \Sigma_{\lambda+c_{0}, \theta_{0}+\epsilon}$ for some small $\epsilon>0$.

Now we recall the properties of the generating function $\delta_{\tau}(\xi)$ and correction term $\mu(\xi)$, which have already been established in [19, eq. (2.13) and Theorem B.1.]. In particular, in case that $z \in \Gamma_{\theta, \sigma}^{\tau}$ there holds that

$$
\begin{array}{ll}
c_{1}|z| \leq\left|\delta_{\tau}\left(e^{-z \tau}\right)\right| \leq c_{2}|z|, & \left|\delta_{\tau}\left(e^{-z \tau}\right)-z\right| \leq c \tau^{k}|z|^{k+1}, \\
\left|\delta_{\tau}\left(e^{-z \tau}\right)^{\alpha}-z^{\alpha}\right| \leq c \tau^{k}|z|^{k+\alpha}, & \left|\mu\left(e^{-z \tau}\right)-1\right| \leq c \tau^{k}|z|^{k} .
\end{array}
$$

To derive an estimate for $w_{n}-w\left(t_{n}\right)$, we compare those two solution representations (3.9) and (3.12). To this end, we use the splitting

$$
\begin{aligned}
u_{n}-u\left(t_{n}\right)= & w_{n}-w\left(t_{n}\right) \\
= & \frac{1}{2 \pi \mathrm{i}} \int_{\Gamma_{\theta, \sigma}^{\tau}} e^{z t_{n}}\left(K\left(\delta_{\tau}\left(e^{-z \tau}\right)\right) \mu\left(e^{-z \tau}\right)-K(z)\right)\left(A u_{0}+g_{0}\right) \mathrm{d} z \\
& -\frac{1}{2 \pi \mathrm{i}} \int_{\Gamma_{\theta, \sigma} \backslash \Gamma_{\theta, \sigma}^{\tau}} e^{z t_{n}} K(z)\left(A u_{0}+g_{0}\right) \mathrm{d} z:=I-I I .
\end{aligned}
$$

Next, we shall bound these two terms separately. By the resolvent estimate (3.4) and approximation properties (3.13), we have

$$
\begin{aligned}
& \quad\left\|\left[K\left(\delta_{\tau}\left(e^{-z \tau}\right)\right)-K(z)\right] \psi\right\|_{L^{\infty}(\Omega)} \\
& \leq\left|\delta_{\tau}\left(e^{-z \tau}\right)^{-1}-z^{-1}\right|\left\|\left(\delta_{\tau}\left(e^{-z \tau}\right)^{\alpha}-A\right)^{-1} \psi\right\|_{L^{\infty}(\Omega)} \\
& \quad+|z|^{-1}\left\|\left[\left(\delta_{\tau}\left(e^{-z \tau}\right)^{\alpha}-A\right)^{-1}-\left(z^{\alpha}-A\right)^{-1}\right] \psi\right\|_{L^{\infty}(\Omega)} \\
& =c \tau^{k}|z|^{k-1}\left\|\left(\delta_{\tau}\left(e^{-z \tau}\right)^{\alpha}-A\right)^{-1} \psi\right\|_{L^{\infty}(\Omega)} \\
& \quad+|z|^{-1}\left|z^{\alpha}-\delta_{\tau}\left(e^{-z \tau}\right)^{\alpha}\right|\left\|\left(\delta_{\tau}\left(e^{-z \tau}\right)^{\alpha}-A\right)^{-1}\left(z^{\alpha}-A\right)^{-1} \psi\right\|_{L^{\infty}(\Omega)} \\
& \leq c \tau^{k}|z|^{k-1}\left|\delta_{\tau}\left(e^{-z \tau}\right)^{\alpha}-\lambda\right|^{-1}\left(1+|z|^{\alpha}\left|z^{\alpha}-\lambda\right|^{-1}\right)\|\psi\|_{L^{\infty}(\Omega)}
\end{aligned}
$$

for $\psi \in C(\bar{\Omega})$. For any $z=\sigma+\rho e^{i \theta}$ with $\rho \leq 1$, we have the uniform bound that

$$
\left|\delta_{\tau}\left(e^{-z \tau}\right)^{\alpha}-\lambda\right|^{-1}+\left|z^{\alpha}-\lambda\right|^{-1} \leq c
$$

since $\delta_{\tau}\left(e^{-z \tau}\right)^{\alpha} \in \Sigma_{\lambda+c_{0}, \theta_{0}+\epsilon}$. Besides, for $z=\sigma+\rho e^{i \theta}$ with $\rho>1$, it holds that

$$
\left|z^{\alpha}-\lambda\right|^{-1} \leq c\left|z^{\alpha}\right|^{-1} \leq c \rho^{-\alpha}
$$

and similarly, using the fact that $\delta_{\tau}\left(e^{-z \tau}\right)^{\alpha} \in \Sigma_{\lambda+c_{0}, \theta_{0}+\epsilon}$ and the approximation properties of generating functions in (3.13), we have for $\rho>1$

$$
\left|\delta_{\tau}\left(e^{-z \tau}\right)^{\alpha}-\lambda\right|^{-1} \leq c\left|\delta_{\tau}\left(e^{-z \tau}\right)^{\alpha}\right|^{-1} \leq c|z|^{-\alpha} \leq c \rho^{-\alpha} .
$$

The same argument also gives the same bound for $z=\sigma+\rho e^{-i \theta}$. Now for the first term in (3.14), we have

$$
\begin{aligned}
\|I\|_{L^{\infty}(\Omega)}= & \left\|\frac{1}{2 \pi \mathrm{i}} \int_{\Gamma_{\theta, \sigma}^{\tau}} e^{z t_{n}}\left(K\left(\delta_{\tau}\left(e^{-z \tau}\right)\right) \mu\left(e^{-z \tau}\right)-K(z)\right)\left(A u_{0}+g_{0}\right) \mathrm{d} z\right\|_{L^{\infty}(\Omega)} \\
\leq & \left\|\frac{1}{2 \pi \mathrm{i}} \int_{\Gamma_{\theta, \sigma}^{\tau}} e^{z t_{n}} K\left(\delta_{\tau}\left(e^{-z \tau}\right)\right)\left(\mu\left(e^{-z \tau}\right)-1\right)\left(A u_{0}+g_{0}\right) \mathrm{d} z\right\|_{L^{\infty}(\Omega)} \\
& +\left\|\frac{1}{2 \pi \mathrm{i}} \int_{\Gamma_{\theta, \sigma}^{\tau}} e^{z t_{n}}\left(K\left(\delta_{\tau}\left(e^{-z \tau}\right)\right)-K(z)\right)\left(A u_{0}+g_{0}\right) \mathrm{d} z\right\|_{L^{\infty}(\Omega)}=: I_{1}+I_{2} .
\end{aligned}
$$


The term $I_{1}$ can be bounded using estimates (3.13), (3.16) and (3.17)

$$
\begin{aligned}
I_{1} & \leq c \tau^{k}\left\|A u_{0}+g_{0}\right\|_{L^{\infty}(\Omega)} \int_{\Gamma_{\theta, \sigma}^{\tau}} e^{\operatorname{Re}(z) t_{n}}|z|^{k-1}\left|z^{\alpha}-\lambda\right|^{-1}|\mathrm{~d} z| \\
& \leq c e^{\sigma t_{n}} \tau^{k}\left\|A u_{0}+g_{0}\right\|_{L^{\infty}(\Omega)}\left(\int_{0}^{1} e^{-c \rho t_{n}} \mathrm{~d} \rho+\int_{1}^{\infty} e^{-c \rho t_{n}} \rho^{k-1-\alpha} \mathrm{d} \rho\right) \\
& \leq c_{T} \tau^{k} t_{n}^{\alpha-k}\left\|A u_{0}+g_{0}\right\|_{L^{\infty}(\Omega)} .
\end{aligned}
$$

Similarly, we apply (3.13)-(3.18) to derive a proper bound for $I_{2}$

$$
\begin{aligned}
I_{2} & \leq c \tau^{k}\left\|A u_{0}+g_{0}\right\|_{L^{\infty}(\Omega)} \int_{\Gamma_{\theta, \sigma}^{\tau}} e^{\operatorname{Re}(z) t_{n}}|z|^{k-1}\left|\delta_{\tau}\left(e^{-z \tau}\right)^{\alpha}-\lambda\right|^{-1}\left(1+|z|^{\alpha}\left|z^{\alpha}-\lambda\right|^{-1}\right)|\mathrm{d} z| \\
& \leq c e^{\sigma t_{n}} \tau^{k}\left\|A u_{0}+g_{0}\right\|_{L^{\infty}(\Omega)}\left(\int_{0}^{1} e^{-c \rho t_{n}} \mathrm{~d} \rho+\int_{1}^{\infty} e^{-c \rho t_{n}} \rho^{k-1-\alpha} \mathrm{d} \rho\right) \\
& \leq c_{T} \tau^{k} t_{n}^{\alpha-k}\left\|A u_{0}+g_{0}\right\|_{L^{\infty}(\Omega)} .
\end{aligned}
$$

Finally, we bound the second term in (3.14) by using the resolvent estimate (3.4):

$$
\begin{aligned}
\|I I\|_{L^{\infty}(\Omega)} & \leq c e^{\sigma t_{n}}\left\|A u_{0}+g_{0}\right\|_{L^{\infty}(\Omega)} \int_{\pi /(\tau \sin \theta)}^{\infty} e^{-c \rho t_{n}} \rho^{-1}\left|\left(\sigma+\rho e^{\mathrm{i} \theta}\right)^{\alpha}-\lambda\right|^{-1} \mathrm{~d} \rho \\
& \leq c_{T} \tau^{k}\left\|A u_{0}+g_{0}\right\|_{L^{\infty}(\Omega)} \int_{\pi /(\tau \sin \theta)}^{\infty} e^{-c \rho t_{n}} \rho^{k-1-\alpha} \mathrm{d} \rho \quad\left(\text { since } 1 \leq \tau^{k}|z|^{k}\right) \\
& \leq c_{T} \tau^{k} t_{n}^{\alpha-k}\left\|A u_{0}+g_{0}\right\|_{L^{\infty}(\Omega)} .
\end{aligned}
$$

This completes the proof of the lemma.

REMARK 3.1. The generic constant $c$ in Lemma 3.1 depends on the terminal time $T$ with $c(T) \sim$ $O\left(e^{\sigma T}\right)$ for some $\sigma>0$. Therefore, the error estimate in Lemma 3.1 is not uniform in $T$ and hence it is not suitable for long-time estimate. This is because the operator $A=\Delta+f^{\prime}\left(u_{0}\right) I$ might not be negative definite, and hence the solution might blow up exponentially as $T \rightarrow \infty$. In case that $A$ is negative definite, we can obtain an error estimate which is uniform in large terminal time (e.g., [19,23]).

Now we turn to the subdiffusion problem driven by a general source term:

$$
\partial_{t}^{\alpha} w(t)-A w(t)=g(t) \quad \text { with } t \in(0, T], \quad \text { and } \quad w(0)=0,
$$

whose time stepping scheme reads

$$
\bar{\partial}_{\tau}^{\alpha} w_{n}-A w_{n}=g_{n}:=g\left(t_{n}\right), \quad 1 \leq n \leq k-1, \quad \text { with } \quad w_{0}=0 .
$$

The time stepping solution $w_{n}$ can be represented by a discrete convolution

$$
w_{n}=\tau \sum_{j=1}^{n} E_{\tau}^{n-j} g_{j}, \text { where } E_{\tau}^{n}=\frac{1}{2 \pi \mathrm{i}} \int_{\Gamma_{\theta, \sigma}^{\tau}} e^{z t_{n}}\left(\delta_{\tau}\left(e^{-z \tau}\right)^{\alpha}+A\right)^{-1} \mathrm{~d} z .
$$

The angle $\theta$ and parameter $\sigma$ are chosen as those in the proof of Lemma 3.1. Then by (3.13), (3.16) and (3.18), we derive that

$$
\begin{aligned}
\left\|E_{\tau}^{n} \psi\right\|_{L^{\infty}(\Omega)} & =\left\|\frac{1}{2 \pi \mathrm{i}} \int_{\Gamma_{\theta, \sigma}^{\tau}} e^{z t_{n}}\left(\delta_{\tau}\left(e^{-z \tau}\right)^{\alpha}+A\right)^{-1} \psi \mathrm{d} z\right\|_{L^{\infty}(\Omega)} \\
& \leq c e^{\sigma T}\left(\int_{1}^{\frac{\pi}{\tau \sin \theta}} e^{-c \rho t_{n}} \rho^{-\alpha} \mathrm{d} \rho+\int_{0}^{1} e^{-c \rho t_{n}} \mathrm{~d} \rho\right) \leq c_{T}\left(t_{n}+\tau\right)^{\alpha-1} .
\end{aligned}
$$

Therefore, it holds the stability that

$$
\left\|w_{n}\right\|_{L^{\infty}(\Omega)} \leq c_{T}\left(\tau \sum_{j=1}^{n} t_{n-j+1}^{\alpha-1}\left\|g_{j}\right\|_{L^{\infty}(\Omega)}\right) .
$$

Here we assume that the source term $g$ satisfies certain compatibility condition, e.g.,

$$
g^{(j)}(0)=0, \quad j=0,1,2, \ldots, k-1 .
$$


For such a source term $g$, by using the resolvent estimates (2.3) and the technique in the proof of Lemma 3.1 the estimate of $w_{n}-w\left(t_{n}\right)$ can be done similarly (hence omitted) as that given in [18, Lemma 3.7], i.e., for all $\ell=1,2, \ldots, k$

$$
\begin{aligned}
\left\|w\left(t_{n}\right)-w_{n}\right\|_{L^{\infty}(\Omega)} & \leq c_{T} \tau^{\ell} \int_{0}^{t_{n}}\left(t_{n}-s\right)^{\alpha-1}\left\|g^{(\ell)}(s)\right\|_{L^{\infty}(\Omega)} \mathrm{d} s \\
& \leq c_{T} \tau^{\ell}\left(t_{n}^{\alpha-1}\|g\|_{W^{\ell, 1}\left(\left(0, t_{n} / 2\right) ; L^{\infty}(\Omega)\right)}+t_{n}^{\alpha}\|g\|_{C^{\ell}\left(\left[t_{n} / 2, t_{n}\right] ; L^{\infty}(\Omega)\right)}\right) .
\end{aligned}
$$

Then by the interpolation, we have the following estimate.

Lemma 3.2. Suppose that $g \in W^{\ell+s, 1}((0, T) ; C(\bar{\Omega})) \cap C^{\ell+1}((0, T) ; C(\bar{\Omega}))$ and $g^{(j)}=0$ with $\ell \in \mathbb{N}^{+}$, $s \in(0,1)$ and $j=0,1, \ldots, \ell$. Let $w(t)$ and $w_{n}$ be the solutions of (3.19) and (3.20), respectively. Then the following error estimate holds

$$
\left\|w\left(t_{n}\right)-w_{n}\right\|_{L^{\infty}(\Omega)} \leq c t_{n}^{\alpha-1} \tau^{\min (k, \ell+s)},
$$

where the constant $c$ depends only on $\alpha, g$ and $T$.

3.3. Error analysis of the BDF scheme for nonlinear problem. Now we turn to the error estimate for the fully implicit scheme (1.8). To this end, we begin with the following lemma, which provides a discrete Hölder bound of the time stepping solution to (1.8).

Lemma 3.3. Assume that the same conditions in Theorem 2.1 holds valid and further $f$ satisfies (3.1). Let $\left\{u_{n}\right\}_{n=1}^{N}$ be the solution to the time stepping scheme (1.8). Then we have

$$
\max _{1 \leq n \leq N}\left\|u_{n}\right\|_{L^{\infty}(\Omega)}+\max _{1 \leq n \leq N} t_{n}^{-\alpha}\left\|u_{n}-u_{0}\right\|_{L^{\infty}(\Omega)} \leq c, \quad \text { for } n=1,2, \ldots, N,
$$

where the constant $c$ depends on $T, u_{0}, f$ but is independent of $\tau$ and $N$.

Proof. By the preceding argument, we have the following representation of $u_{n}$ :

$$
u_{n}=u_{0}+F_{\tau}^{n}\left(A u_{0}+g_{0}\right)+\tau \sum_{j=1}^{n} E_{\tau}^{n-j} R\left(u_{j} ; u_{0}\right)
$$

as well as the bound that (by Theorem 3.1 and the estimate (3.22)

$$
\left\|F_{\tau}^{n}\right\|_{C(\bar{\Omega}) \rightarrow C(\bar{\Omega})} \leq c_{T} t_{n}^{\alpha} \quad \text { and } \quad\left\|E_{\tau}^{n}\right\|_{C(\bar{\Omega}) \rightarrow C(\bar{\Omega})} \leq c_{T} t_{n}^{\alpha-1} .
$$

Therefore, by the Lipchitz continuity of the modified potential term $\bar{f}(s)$, we have

$$
\begin{aligned}
\left\|u_{n}-u_{0}\right\|_{L^{\infty}(\Omega)} & \leq c_{T} t_{n}^{\alpha}+\tau \sum_{j=1}^{n} t_{n-j+1}^{\alpha-1}\left\|R\left(u_{j} ; u_{0}\right)\right\|_{L^{\infty}(\Omega)} \\
& \leq c_{T} t_{n}^{\alpha}+\tau \sum_{j=1}^{n} t_{n-j+1}^{\alpha-1}\left(\left\|f\left(u_{j}\right)-f\left(u_{0}\right)\right\|_{L^{\infty}(\Omega)}+\left\|f^{\prime}\left(u_{0}\right)\left(u_{j}-u_{0}\right)\right\|_{L^{\infty}(\Omega)}\right) \\
& \leq c_{T} t_{n}^{\alpha}+\tau \sum_{j=1}^{n} t_{n-j+1}^{\alpha-1}\left\|u_{j}-u_{0}\right\|_{L^{\infty}(\Omega)} .
\end{aligned}
$$

Then by the discrete Grönwall's inequality [11, Lemma 7.1], we obtain that

$$
\left\|u_{n}-u_{0}\right\|_{L^{\infty}(\Omega)} \leq c t_{n}^{\alpha}
$$

where the constant $c$ depends on $T, u_{0}, f$, but it is independent of $\tau$ and $N$. Finally, the uniform bound of $\left\|u_{n}\right\|_{L^{\infty}(\Omega)}$ follows from the triangle inequality. $\square$

Now we are ready to state our main theorem in the section.

THEOREM 3.4. Assume that the same conditions in Theorem[2.1 holds valid and further $f$ satisfies (3.1). Let $u(t)$ be the solution of the semilinear subdiffusion problem (1.1) and $\left\{u_{n}\right\}_{n=1}^{N}$ be the solution of fully implicit scheme (1.8). Then the following error estimate holds

$$
\left\|u_{n}-u\left(t_{n}\right)\right\|_{L^{\infty}(\Omega)} \leq c \tau^{\min (k, 1+2 \alpha-\epsilon)} t_{n}^{\alpha-\min (k, 1+2 \alpha-\epsilon)},
$$

for any $t_{n}>0$ and arbitrarily small $\epsilon>0$. Here the constant $c$ depends on $T, u_{0}, f, \epsilon$, but it is 
independent of $\tau$ and $N$.

Proof. To begin with, we split the solution $u(t)$ into two components

$$
u(t)=v(t)+w(t),
$$

where $v$ is the solution of (3.6), and $w$ satisfies (3.19) with $g=R\left(u ; u_{0}\right)$. Similarly, the time stepping solution can also be separated by

$$
u_{n}=v_{n}+w_{n},
$$

where $v_{n}$ is the solution of (3.7), and $w_{n}$ satisfies (3.20) with $g_{n}=R\left(u_{n} ; u_{0}\right)$, i.e., by (3.21)

$$
w_{n}=\tau \sum_{j=1}^{n} E_{\tau}^{n-j} R\left(u_{j} ; u_{0}\right)
$$

We note that the difference between $v\left(t_{n}\right)$ and $v_{n}$ has been estimated in Lemma 3.1. In order to study $w_{n}-w\left(t_{n}\right)$, we use an intermediate solution $\bar{w}_{n}$ which satisfies (3.20) with $g_{n}=R\left(u\left(t_{n}\right) ; u_{0}\right)$ and can be represented by

$$
\bar{w}_{n}=\tau \sum_{j=1}^{n} E_{\tau}^{n-j} R\left(u\left(t_{j}\right) ; u_{0}\right)
$$

Then the regularity of $R\left(u ; u_{0}\right)$ proved in Theorem 2.2 and the estimate in Lemma 3.2 yield

$$
\left\|\bar{w}_{n}-w\left(t_{n}\right)\right\|_{L^{\infty}(\Omega)} \leq c \tau^{1+2 \alpha-\epsilon} t_{n}^{\epsilon-\alpha-1} .
$$

To sum up, we derive a bound of $e_{n}=u_{n}-u\left(t_{n}\right)$ :

$$
\begin{aligned}
\left\|e_{n}\right\|_{L^{\infty}(\Omega)} & =\left\|v_{n}-v\left(t_{n}\right)\right\|_{L^{\infty}(\Omega)}+\left\|\bar{w}_{n}-w\left(t_{n}\right)\right\|_{L^{\infty}(\Omega)}+\left\|w_{n}-\bar{w}_{n}\right\|_{L^{\infty}(\Omega)} \\
& \leq c \tau^{k} t_{n}^{\alpha-k}+c \tau^{1+2 \alpha-\epsilon} t_{n}^{\alpha-1}+\tau \sum_{j=1}^{n}\left\|E_{\tau}^{n-j}\left[R\left(u_{j} ; u_{0}\right)-R\left(u\left(t_{j}\right) ; u_{0}\right)\right]\right\|_{L^{\infty}(\Omega)} \\
& \leq c \tau^{\min (k, 1+2 \alpha-\epsilon)} t_{n}^{\alpha-\min (k, 1+2 \alpha-\epsilon)}+c \tau \sum_{j=1}^{n} t_{n-j+1}^{\alpha-1}\left\|R\left(u_{j} ; u_{0}\right)-R\left(u\left(t_{j}\right) ; u_{0}\right)\right\|_{L^{\infty}(\Omega)} .
\end{aligned}
$$

Recalling Lemma 3.3 and the fact that $u \in C^{\alpha}([0, T] ; C(\bar{\Omega}))$, we obtain that

$$
\begin{aligned}
& \left\|R\left(u_{j} ; u_{0}\right)-R\left(u\left(t_{j}\right) ; u_{0}\right)\right\|_{L^{\infty}(\Omega)} \\
\leq & \left\|\int_{u_{0}}^{u\left(t_{j}\right)}\left(u\left(t_{j}\right)-u_{j}\right) f^{\prime \prime}(s) d s\right\|_{L^{\infty}(\Omega)}+\left\|\int_{u\left(t_{j}\right)}^{u_{j}}\left(u_{j}-s\right) f^{\prime \prime}(s) d s\right\|_{L^{\infty}(\Omega)} \\
\leq & c\left(\left\|u_{j}-u_{0}\right\|_{L^{\infty}(\Omega)}+\left\|u\left(t_{j}\right)-u_{0}\right\|_{L^{\infty}(\Omega)}\right)\left\|e_{n}\right\|_{L^{\infty}(\Omega)} \\
\leq & c t_{n}^{\alpha}\left\|e_{n}\right\|_{L^{\infty}(\Omega)},
\end{aligned}
$$

and hence we arrive at the estimate

$$
\left\|e_{n}\right\|_{L^{\infty}(\Omega)} \leq c \tau^{\min (k, 1+2 \alpha-\epsilon)} t_{n}^{\alpha-\min (k, 1+2 \alpha-\epsilon)}+c \tau \sum_{j=1}^{n} t_{n-j+1}^{\alpha-1} t_{j}^{\alpha}\left\|e_{j}\right\|_{L^{\infty}(\Omega)} .
$$

After multiplying $t_{n}^{\alpha}$ on both sides, we have

$$
t_{n}^{\alpha}\left\|e_{n}\right\|_{L^{\infty}(\Omega)} \leq c \tau^{\min (k, 1+2 \alpha-\epsilon)} t_{n}^{2 \alpha-\min (k, 1+2 \alpha-\epsilon)}+c t_{n}^{\alpha} \tau \sum_{j=1}^{n} t_{n-j+1}^{\alpha-1} t_{j}^{\alpha}\left\|e_{j}\right\|_{L^{\infty}(\Omega)} .
$$

Noting that $2 \alpha-\min (k, 1+2 \alpha-\epsilon)>-1$, we apply the Grönwall's inequality [11, Lemma 7.1] for $t_{n}^{\alpha}\left\|e_{n}\right\|_{L^{\infty}(\Omega)}$ and derive that

$$
t_{n}^{\alpha}\left\|e_{n}\right\|_{L^{\infty}(\Omega)} \leq c \tau^{\min (k, 1+2 \alpha-\epsilon)} t_{n}^{2 \alpha-\min (k, 1+2 \alpha-\epsilon)} .
$$

This completes the proof. 
REMARK 3.2. The result in Theorem 3.4 implies a uniform-in-time error

$$
\max _{1 \leq n \leq N}\left\|u_{n}-u\left(t_{n}\right)\right\|_{L^{\infty}(\Omega)} \leq c \tau^{\alpha-\epsilon},
$$

for some small $\epsilon>0$. This result is consistent with the error estimate in [20].

REMARK 3.3. The error estimate in Theorem 3.4 indicates that the best convergence rate of the corrected BDFk scheme (1.8) is almost of order $O\left(\tau^{\min (k, 1+2 \alpha)}\right)$, due to the low regularity of the remainder $R\left(u ; u_{0}\right)$ (see Theorem 2.2 and Lemma 3.2). The reason is that $u$ is nonsmooth in the time direction, even though the initial condition is smooth and compatible with the boundary condition. This phenomena contrasts sharply with its normal parabolic counterpart, i.e., $\alpha=1$. For instance, it has been proved in [7] that the time stepping schemes of the semilinear parabolic equation are able to achieve a better convergence rate in case of regular initial data.

3.4. Numerical analysis without globally Lipschitz condition. The preceding analysis could be easily extended to the nonlinear subdiffusion problem without the globally Lipschitz condition (3.1). For completeness, we briefly sketch the argument in this section.

Under the assumptions in Theorem 2.1 and letting

$$
b=\|u\|_{L^{\infty}((0, T) \times \Omega)}+1,
$$

we are able to define a smooth function $\bar{f}$ such that

$$
\bar{f}(s)=f(s) \quad \text { for all }-b \leq s \leq b,
$$

and it is globally Lipschitz continuous

$$
|\bar{f}(s)-\bar{f}(t)| \leq c_{L}|t-s| \quad \text { for all } t, s \in \mathbb{R} .
$$

Then we consider the BDF scheme with potential term $\bar{f}$ instead of $f$

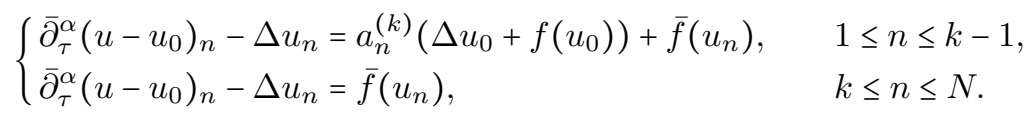

Then under the condition (3.27) we know that (3.28) admits a unique solution. Meanwhile, Theorem 3.4 indicates a uniform-in-time error estimate

$$
\max _{1 \leq n \leq N}\left\|u_{n}-u\left(t_{n}\right)\right\|_{L^{\infty}(\Omega)} \leq c \tau^{\alpha-\epsilon},
$$

for some small $\epsilon>0$, where the constant $c$ depends on $T, u_{0}, \bar{f}, \epsilon$.

As a result, for $\tau<\tau_{0}$ such that $c \tau_{0}^{\alpha-\epsilon}=1$, we have

$$
\max _{1 \leq n \leq N}\left\|u_{n}\right\|_{L^{\infty}(\Omega)} \leq \max _{1 \leq n \leq N}\left\|u\left(t_{n}\right)\right\|_{L^{\infty}(\Omega)}+c \tau^{\alpha-\epsilon} \leq b+1 .
$$

Therefore $\bar{f}\left(u_{n}\right)=f\left(u_{n}\right)$ for all $1 \leq n \leq N$, and the modified time stepping scheme (3.28) is identical to the original one (1.9) (or equivalently (1.8)). Then we have the following corollary.

Corollary 3.5. Assume that the same conditions in Theorem 2.1 hold valid. Let $u(t)$ be the solution of the semilinear subdiffusion problem (1.1) and $\left\{u_{n}\right\}_{n=1}^{N}$ be the solution of fully implicit scheme (1.8). Then the following error estimate holds

$$
\left\|u_{n}-u\left(t_{n}\right)\right\|_{L^{\infty}(\Omega)} \leq c \tau^{\min (k, 1+2 \alpha-\epsilon)} t_{n}^{\alpha-\min (k, 1+2 \alpha-\epsilon)},
$$

for any $t_{n}>0$ and arbitrarily small $\epsilon>0$. Here the constant $c$ depends on $T, u_{0}, f, \epsilon$, but it is independent of $\tau$ and $N$.

REMARK 3.4. In [8], Cuesta et. al studied a second-order BDF method for solving a related (but different) subdiffusion model

$$
u-\partial_{t}^{-\alpha} \Delta u=u_{0}+\partial_{t}^{-1} f(u) \text { with } u(0)=u_{0},
$$


under the assumption that $f$ is sufficiently smooth and the solution $u$ can be expanded as

$$
u(t)=\sum_{m, l \geq 0 ; m+l \alpha<2} c_{m, l} t^{m+l \alpha}+v(t) \quad \text { with } \quad c_{m l} \in D(\Delta) \quad \text { and } \quad v \in C^{2}([0, T] ; D(\Delta)) .
$$

This assumption requires stronger compatibility conditions of $u_{0}$ and $f\left(u_{0}\right)$. As a simple example, we consider the homogeneous problem, i.e. $f \equiv 0$. In this case, the solution of the subdiffusion problem (1.1) can be expanded by Mittag-Leffler function as

$$
u(t)=E_{\alpha, 1}\left(\Delta t^{\alpha}\right) u_{0}=\sum_{k=0}^{\infty} \frac{1}{\Gamma(\alpha k+1)}\left((\Delta)^{k} u_{0}\right) t^{\alpha k} .
$$

Then assumption (3.30) requires that $u_{0} \in D\left((-\Delta)^{1+2 / \alpha}\right)$ which is stronger than what we assumed in this work.

4. Fully discrete scheme and error analysis. In this section, we will briefly discuss the fully discrete scheme for solving the nonlinear subdiffusion equation (1.1). We shall start with a spatially semidiscrete scheme for problem (1.1) based on the standard Galerkin finite element method (see e.g., 14,17] for linear subdiffusion problems).

For $h \in\left(0, h_{0}\right], h_{0}>0$, we denote by $\mathcal{T}_{h}=\left\{K_{j}\right\}$ a triangulation of $\Omega_{h}=\operatorname{Int}\left(\cup \bar{K}_{j}\right)$ into mutually disjoint open face-to-face simplices $K_{j}$. Assume that all vertices of a simplex $K_{j}$ locate on $\partial \Omega$. We also assume that $\left\{\mathcal{T}_{h}\right\}$ is globally quasi-uniform, i.e., $\left|K_{j}\right| \geq c h^{d}$ with a given $c>0$. Let $X_{h}$ be the finite dimensional space of continuous piecewise linear functions associated with $\mathcal{T}_{h}$, that vanish outside $\Omega_{h}$. Then we define the $L^{2}(\Omega)$ projection $P_{h}: L^{2}(\Omega) \rightarrow X_{h}$ and Ritz projection $R_{h}: H_{0}^{1} \rightarrow X_{h}$ respectively by

$$
\begin{aligned}
\left(P_{h} \varphi, v_{h}\right) & =\left(\varphi, v_{h}\right), \quad \forall v_{h} \in X_{h}, \\
\left(\nabla R_{h} v, \nabla v_{h}\right) & =\left(\nabla v, \nabla v_{h}\right), \quad \forall v_{h} \in X_{h} .
\end{aligned}
$$

The semidiscrete scheme reads: find $u_{h}(t) \in X_{h}$ such that

$$
\left(\partial_{t}^{\alpha} u_{h}(t), v_{h}\right)+\left(\nabla u_{h}(t), \nabla v_{h}\right)=\left(f\left(u_{h}(t)\right), v_{h}\right) \text { for all } v_{h} \in X_{h},
$$

with $u_{h}(t)=R_{h} u_{0}$. Let $\Delta_{h}: X_{h} \rightarrow X_{h}$ denote the Galerkin finite element approximation of the Dirichlet Laplacian $\Delta$, defined by

$$
\left(\Delta_{h} w_{h}, v_{h}\right):=-\left(\nabla w_{h}, \nabla v_{h}\right), \quad \forall w_{h}, v_{h} \in X_{h} .
$$

Then the spatially semidiscrete scheme (4.1) could be written as

$$
\partial_{t}^{\alpha} u_{h}(t)-\Delta_{h} u_{h}(t)=P_{h} f\left(u_{h}\right), \quad \text { with } u_{h}(0)=R_{h} u_{0} .
$$

With the Laplace transform and convolution rule, $u_{h}(t)$ can be explicitly expressed by

$$
u(t)=\left(I+F_{h}(t) \Delta_{h}\right) u_{0}+\int_{0}^{t} E_{h}(t-s) f(u(s)) \mathrm{d} s,
$$

where the operators $F_{h}(t)$ and $E_{h}(t)$ are defined by

$$
F_{h}(t)=\frac{1}{2 \pi \mathrm{i}} \int_{\Gamma_{\theta, \delta}} e^{z t} z^{-1}\left(z^{\alpha}-\Delta_{h}\right)^{-1} \mathrm{~d} z \quad \text { and } \quad E_{h}(t)=\frac{1}{2 \pi \mathrm{i}} \int_{\Gamma_{\theta, \delta}} e^{z t}\left(z^{\alpha}-\Delta_{h}\right)^{-1} \mathrm{~d} z,
$$

respectively. Recall that the discrete Laplacian satisfies the resolvent estimate in $L^{\infty}(\Omega)$ sense (cf. 4, Theorem 1.1]), i.e., for any angle $\phi \in(\pi / 2, \pi)$,

$$
\left\|\left(z-\Delta_{h}\right)^{-1} w_{h}\right\|_{L^{\infty}(\Omega)} \leq c|z|^{-1}\left\|w_{h}\right\|_{L^{\infty}(\Omega)} \quad \forall z \in \Sigma_{\phi}
$$

This immediately implies the following smoothing properties:

$$
\left\|F_{h} \Delta_{h} v_{h}\right\|_{L^{\infty}(\Omega)}+t^{1-\alpha}\left\|E_{h} v_{h}\right\|_{L^{\infty}(\Omega)}+t\left\|E_{h} \Delta_{h} v_{h}\right\|_{L^{\infty}(\Omega)} \leq c\left\|v_{h}\right\|_{L^{\infty}(\Omega)} \quad \forall v_{h} \in X_{h},
$$

which plays an important role in error analysis. Note that the $L^{\infty}(\Omega)$-norm error analysis of the scheme (4.2) remains scarce, even though the $L^{2}(\Omega)$-norm estimate has been completely understood (cf. [1,20]). For completeness, we shall provide an error estimate in $L^{\infty}(\Omega)$-norm. 
4.1. Spatially semidiscrete scheme for the linear problem. First we recall some error estimates for the following linear subdiffusion equation:

$$
\partial_{t}^{\alpha} v(t)-\Delta v(t)=g(t), \quad \forall t \in(0, T],
$$

where $g$ is a given source function, and $v(0) \in D$ is the given initial condition. The semidiscrete FEM for (4.7) seeks $v_{h}(t) \in X_{h}$ such that

$$
\partial_{t}^{\alpha} v_{h}(t)-\Delta_{h} v_{h}(t)=P_{h} g(t), \quad \forall t \in(0, T]
$$

with $v_{h}(0)=R_{h} v(0)$. Recall that $R_{h}$ has the almost stability property [41, eq. (6.60)]

$$
\left\|R_{h} w\right\|_{L^{\infty}(\Omega)} \leq c \ell_{h}\|w\|_{L^{\infty}(\Omega)}, \quad \text { with } \ell_{h}=\max (1, \log (1 / h)) .
$$

To derive the error estimate of (4.7), we need the following lemma for the Ritz projection $R_{h}$, where the proof relies on the smoothing property of the solution operator $F(t)$ :

$$
\|\Delta F(t) w\|_{L^{p}(\Omega)} \leq c\|w\|_{L^{p}(\Omega)}, \quad \text { for all } p \in[1, \infty) .
$$

This follows directly from the representation (2.2) and the resolvent estimate [35. Theorem 3.1]

$$
\left\|(z-\Delta)^{-1} w\right\|_{L^{p}(\Omega)} \leq c_{p}|z|^{-1}\|w\|_{L^{p}(\Omega)} \quad \forall z \in \Sigma_{\phi}, \phi \in(\pi / 2, \pi), p \in[1, \infty) .
$$

LEMMA 4.1. Let $v$ be the solution of the linear problem (4.7). Then there holds

$$
\left\|\left(v-R_{h} v\right)(t)\right\|_{L^{\infty}(\Omega)} \leq c h^{2} \ell_{h}^{2}\left(\|\Delta v(0)\|_{L^{\infty}(\Omega)}+\int_{0}^{t}\left\|g^{\prime}(s)\right\|_{L^{\infty}(\Omega)} d s\right)
$$

with $\ell_{h}=\max (1, \log (1 / h))$.

Proof. Let $I_{h}$ be the Lagrange interpolation operator. Then we have

$$
v-R_{h} v=\left(R_{h}-I\right)\left(v-I_{h} v\right)
$$

and hence by (4.9) and the approximation property of $I_{h}$, we derive for $2 \leq p<\infty$

$$
\left\|v-R_{h} v\right\|_{L^{\infty}} \leq c \ell_{h}\left\|v-I_{h} v\right\|_{L^{\infty}(\Omega)} \leq c h^{2-2 / p} \ell_{h}\|v\|_{W^{2, p}(\Omega)} .
$$

Now using the full elliptic regulariy, we have for $2 \leq p<\infty$ [41, eq. (6.78)]

$$
\|v\|_{W^{2, p}(\Omega)} \leq c p\|\Delta v\|_{L^{p}(\Omega)} .
$$

Recalling the solution representation (2.1), we have

$$
\begin{aligned}
\Delta v & =\Delta(I+F(t) \Delta) v(0)+\int_{0}^{t} \Delta E(t-s) g(s) \mathrm{d} s \\
& =\Delta(I+F(t) \Delta) v(0)+\int_{0}^{t} \Delta F(t-s) g^{\prime}(s) \mathrm{d} s-\Delta(F(0) g(t)-F(t) g(0))
\end{aligned}
$$

Now we apply the smoothing property (4.10) and arrive at

$$
\|\Delta v\|_{L^{p}(\Omega)} \leq c\|\Delta v(0)\|_{L^{p}(\Omega)}+c \int_{0}^{t}\left\|g^{\prime}(s)\right\|_{L^{p}(\Omega)} d s .
$$

Then the desired result follows immediately by choosing $p=\ell_{h}$.

The semidiscrete solution $v_{h}$ satisfies the following error estimate.

LEMMA 4.2 (Semidiscrete solution of linear problems). For the semidiscrete solution $v_{h}$ to problem (4.8), there holds, with $\ell_{h}=\max (1, \log (1 / h))$, that

$$
\max _{t \in[0, T]}\left\|v_{h}(t)-v(t)\right\|_{L^{2}(\Omega)} \leq c h^{2} \ell_{h}^{3}\left(\|\Delta v(0)\|_{L^{\infty}(\Omega)}+\int_{0}^{t}\left\|g^{\prime}(s)\right\|_{L^{\infty}(\Omega)} d s\right) .
$$

Proof. We use the splitting $v_{h}-v=\left(v_{h}-P_{h} v\right)+\left(P_{h} v-v\right)=: \psi+\theta$. By Lemma 4.1] and [9, Corollary], it is easy to see for all $t \in[0, T]$

$$
\|\theta(t)\|_{L^{\infty}(\Omega)}+\left\|\left(P_{h} v-R_{h} v\right)(t)\right\|_{L^{\infty}(\Omega)} \leq c h^{2} \ell_{h}^{2}\left(\|\Delta v(0)\|_{L^{\infty}(\Omega)}+\int_{0}^{t}\left\|g^{\prime}(s)\right\|_{L^{\infty}(\Omega)} \mathrm{d} s\right) .
$$


Besides, we note that $\psi$ satisfies the equation

$$
\partial_{t}^{\alpha} \psi(t)-\Delta_{h} \psi(t)=\Delta_{h}\left(R_{h}-P_{h}\right) v(t), \quad \text { with } \psi(0)=\left(R_{h}-P_{h}\right) v .
$$

Therefore, by the representation (4.3), we arrive at

$$
\psi(t)=\left(I+F_{h}(t) \Delta_{h}\right)\left(R_{h}-P_{h}\right) v(0)+\int_{0}^{t} E_{h}(t-s) \Delta_{h}\left(R_{h}-P_{h}\right) v(s) d s=: I_{1}+I_{2} .
$$

The estimate of $I_{1}$ follows directly from (4.6) and (4.11)

$$
\left\|I_{1}\right\|_{L^{\infty}(\Omega)} \leq c\left\|\left(R_{h}-P_{h}\right) v(0)\right\|_{L^{\infty}(\Omega)} \leq c h^{2} \ell_{h}^{2}\|\Delta v(0)\|_{L^{\infty}(\Omega)} .
$$

For the second term, we apply the inverse inequality for finite element functions, as well as (4.6) and (4.11), to obtain that

$$
\begin{aligned}
\left\|I_{2}\right\|_{L^{\infty}(\Omega)} & \leq c h^{-2 \epsilon} \int_{0}^{t}(t-s)^{-1+\epsilon}\left\|\left(R_{h}-P_{h}\right) v(s)\right\|_{L^{\infty}(\Omega)} \mathrm{d} s \\
& \leq c \epsilon^{-1} h^{2-2 \epsilon} \ell_{h}^{2}\left(\|\Delta v(0)\|_{L^{\infty}(\Omega)}+\int_{0}^{t}\left\|g^{\prime}(s)\right\|_{L^{\infty}(\Omega)} \mathrm{d} s\right)
\end{aligned}
$$

by choosing $\epsilon=1 / \ell_{h}$, then we complete the proof of the lemma.

4.2. Error analysis for the nonlinear problem. Now we turn to the nonlinear problem (1.1). The following lemma provides an error estimate of the semidiscrete scheme (4.2).

Lemma 4.3. Assume that the same conditions in Theorem 2.1 hold valid. Then the semidiscrete problem (4.2) has a unique solution $u_{h} \in C([0, T] \times \bar{\Omega})$, which satisfies

$$
\max _{0 \leq t \leq T}\left\|u(t)-u_{h}(t)\right\|_{L^{\infty}(\Omega)} \leq c h^{2} \ell_{h}^{3}, \quad \text { with } \ell_{h}=\max (1, \log (1 / h)) .
$$

Proof. To begin with, we assume that the nonlinear term $f: \mathbb{R} \rightarrow \mathbb{R}$ is Globally Lipschitz continuous. Then, by the argument in [20, Theorem 3.1], the existence and uniqueness of the solution $u_{h}$ hold. It remains to establish the estimate (4.12). To this end, we define $v_{h}(t)$ as the solution of

$$
\partial_{t}^{\alpha} v_{h}(t)-\Delta_{h} v_{h}(t)=P_{h} f(u(t)), \quad \text { with } \quad v_{h}(0)=R_{h} u_{0} .
$$

This together with Lemma 4.2 and Theorem 2.1 yields the following estimate for $t \geq 0$

$$
\left\|\left(u-v_{h}\right)(t)\right\|_{L^{2}(\Omega)} \leq c h^{2} \ell_{h}^{3} .
$$

Meanwhile, we note that $\rho_{h}:=v_{h}-u_{h}$ satisfies the following equation

$$
\partial_{t}^{\alpha} \rho_{h}(t)-\Delta \rho_{h}(t)=P_{h} f(u(t))-P_{h} f\left(u_{h}(t)\right), \quad \text { with } \quad \rho_{h}(0)=0 .
$$

Then, by the smoothing property (4.6), the Lipschitz continuity of $f$ and the stability of $P_{h}$ in $L^{\infty}(\Omega)$ 9], we derive that

$$
\begin{aligned}
\left\|\rho_{h}(t)\right\|_{L^{\infty}} & \leq \int_{0}^{t}\left\|E_{h}(t-s) P_{h}\left[f(u(s))-f\left(u_{h}(s)\right)\right]\right\|_{L^{\infty}(\Omega)} \mathrm{d} s \\
& \leq c \int_{0}^{t}(t-s)^{\alpha-1}\left\|P_{h}\left[f(u(s))-f\left(u_{h}(s)\right)\right]\right\|_{L^{\infty}(\Omega)} \mathrm{d} s \\
& \leq c \int_{0}^{t}(t-s)^{\alpha-1}\left\|u(s)-u_{h}(s)\right\|_{L^{\infty}(\Omega)} \mathrm{d} s \\
& \leq c h^{2} \ell_{h}^{3}+c \int_{0}^{t}(t-s)^{\alpha-1}\left\|\rho_{h}(s)\right\|_{L^{\infty}(\Omega)} \mathrm{d} s .
\end{aligned}
$$

Then by the Grönwall's inequality, we have

$$
\max _{t \in[0, T]}\left\|\rho_{h}(t)\right\|_{L^{2}(\Omega)} \leq c h^{2} \ell_{h}^{3} .
$$

This and (4.13) directly imply the desired result. Then the same argument as the one in Section 3.4 helps to remove the the globally Lipschitz condition. 
Finally, we consider the fully discrete scheme: find $U_{h}^{n}$ such that

$$
\left\{\begin{array}{lll}
\bar{\partial}_{\tau}^{\alpha}\left(U_{h}^{n}-U_{h}^{0}\right)-\Delta_{h} U_{h}^{n}=a_{n}^{(k)}\left(\Delta_{h} U_{h}^{n}+f\left(U_{h}^{0}\right)+f\left(U_{h}^{0}\right),\right. & & 1 \leq n \leq k-1, \\
\bar{\partial}_{\tau}^{\alpha}\left(U_{h}^{n}-U_{h}^{0}\right)-\Delta_{h} U_{h}^{n}=f\left(U_{h}^{n}\right), & & k \leq n \leq N .
\end{array}\right.
$$

Then by the resolvent estimate (4.5), all the arguments in Sections 2 and 3 work for the spatially discrete problems (4.2) and (4.14). Therefore we have the following corollary.

Corollary 4.4. Assume that the same conditions in Theorem 2.1 hold valid. Let $u_{h}(t)$ be the solution of the semidiscrete scheme (4.2) and $\left\{U_{h}^{n}\right\}_{n=1}^{N}$ be the solution of fully discrete scheme (4.14). Then the following error estimate holds

$$
\left\|U_{h}^{n}-u_{h}\left(t_{n}\right)\right\|_{L^{\infty}(\Omega)} \leq c \tau^{\min (k, 1+2 \alpha-\epsilon)} t_{n}^{\alpha-\min (k, 1+2 \alpha-\epsilon)},
$$

for any $t_{n}>0$ and arbitrarily small $\epsilon>0$. Here the constant $c$ depends on $T, u_{0}, f, \epsilon$, but it is independent of $h, \tau$ and $N$.

This corollary together with Lemma 4.3 immediately leads to the error estimate of the fully discrete scheme (4.14).

TheOREM 4.5. Assume that the same conditions in Theorem [2.1 hold valid. Let $u(t)$ be the solution of the semilinear subdiffusion problem (1.1) and $\left\{U_{h}^{n}\right\}_{n=1}^{N}$ be the solution of fully discrete scheme (4.14). Then for $\ell_{h}=\max (1, \log (1 / h))$, the following error estimate holds

$$
\left\|U_{h}^{n}-u\left(t_{n}\right)\right\|_{L^{\infty}(\Omega)} \leq c h^{2} \ell_{h}^{3}+\tau^{\min (k, 1+2 \alpha-\epsilon)} t_{n}^{\alpha-\min (k, 1+2 \alpha-\epsilon)},
$$

for any $t_{n}>0$ and arbitrarily small $\epsilon>0$. The constant $c$ depends on $T, u_{0}, f, \epsilon$, but it is independent of $h, \tau$ and $N$.

5. Numerical experiments. In this section, we present numerical results to illustrate and support our theoretical findings. We consider the nonlinear subdiffusion model with $\Omega=(0,1)^{2}$

$$
\left\{\begin{aligned}
\partial_{t}^{\alpha} u-\frac{1}{10} \Delta u & =4\left(u-u^{3}\right) & & \text { in } \Omega \times(0, T), \\
u & =0 & & \text { on } \partial \Omega \times(0, T), \\
u(0) & =u_{0} & & \text { in } \Omega,
\end{aligned}\right.
$$

In the computation, we divided the domain $\Omega$ into regular right triangles with $M$ equal subintervals of length $h$ on each side of the domain. The numerical solutions are computed by using fully discrete scheme (4.14). In each step, we solved the nonlinear elliptic problem by Newton's iteration. We fixed the spatial mesh size $h=1 / 100$, computed the numerical solution $\left\{U_{h}^{N}\right\}$ with temporal step size $\tau=T / N$ with $T=1, N=100 \times 2^{\ell}, \ell=0,1, \ldots, 4$ and reported

$$
e_{\tau}=\left\|U_{h}^{N}-u_{h}\left(t_{N}\right)\right\|_{L^{\infty}(\Omega)} .
$$

Since the semidiscrete solution $u_{h}$ is unavailable, we compute reference solutions on a finer mesh, i.e., the fully discrete solution $U_{h}^{N}$ with $h=1 / 100, N=20000$ and $k=6$.

We consider the following problem data:

$$
u_{0}(x, y)=4 x(1-x) y(1-y)
$$

where the initial condition satisfies

$$
u_{0}, \Delta u_{0} \in C(\bar{\Omega}) \quad \text { and } \quad u=0 \text { on } \partial \Omega .
$$

Therefore, our assumptions on initial condition (i.e., $u_{0} \in D$ ) are fulfilled. In Table 2, we present numerical results of the corrected $k$-step BDF scheme (1.8). Numbers in brackets are the theoretical convergence rates. Numerical results show that the convergence rate is $O\left(\tau^{\min (k, 1+2 \alpha)}\right)$. For example, in case that $\alpha=0.7$, we observe an $O\left(\tau^{2.4}\right)$ rate of BDF $k$ scheme with $k=3,4,5,6$, but an $O\left(\tau^{2}\right)$ rate in case that $k=2$. This is in good agreement with our theoretical results. In Table 3 , we present numerical results for uncorrected $k$-step BDF schemes (1.2). We observe that all schemes are 
first-order accurate. This phenomena has already been reported for the linear fractional evolution equations [16, 19]. This implies the necessity of the modification in the starting steps.

TABLE 2

Corrected BDFk scheme (1.8) at $T=1$ with $h=1 / 100$ and $\tau=1 /\left(100 \times 2^{\ell}\right)$

\begin{tabular}{|c|c|ccccc|c|}
\hline$\alpha$ & $k \backslash \ell$ & 0 & 1 & 2 & 3 & 4 & rate \\
\hline \multirow{5}{*}{0.3} & $k=2$ & $2.94 \mathrm{e}-06$ & $9.99 \mathrm{e}-07$ & $3.45 \mathrm{e}-07$ & $1.20 \mathrm{e}-07$ & $4.19 \mathrm{e}-08$ & $\approx 1.52(1.60)$ \\
& $k=3$ & $2.43 \mathrm{e}-06$ & $8.90 \mathrm{e}-07$ & $3.21 \mathrm{e}-07$ & $1.14 \mathrm{e}-07$ & $3.99 \mathrm{e}-08$ & $\approx 1.51(1.60)$ \\
& $k=4$ & $4.36 \mathrm{e}-06$ & $1.57 \mathrm{e}-06$ & $5.58 \mathrm{e}-07$ & $1.96 \mathrm{e}-07$ & $6.82 \mathrm{e}-08$ & $\approx 1.52(1.60)$ \\
& $k=5$ & $9.73 \mathrm{e}-06$ & $3.45 \mathrm{e}-06$ & $1.21 \mathrm{e}-06$ & $4.23 \mathrm{e}-07$ & $1.46 \mathrm{e}-07$ & $\approx 1.53(1.60)$ \\
& $k=6$ & $5.17 \mathrm{e}-09$ & $1.70 \mathrm{e}-09$ & $5.60 \mathrm{e}-10$ & $1.85 \mathrm{e}-10$ & $6.09 \mathrm{e}-11$ & $\approx 1.60(1.60)$ \\
\hline \multirow{5}{*}{0.5} & $k=2$ & $2.79 \mathrm{e}-06$ & $7.53 \mathrm{e}-07$ & $2.02 \mathrm{e}-07$ & $5.44 \mathrm{e}-08$ & $1.45 \mathrm{e}-08$ & $\approx 1.91(2.00)$ \\
& $k=3$ & $6.42 \mathrm{e}-07$ & $1.75 \mathrm{e}-07$ & $4.63 \mathrm{e}-08$ & $1.20 \mathrm{e}-08$ & $3.07 \mathrm{e}-09$ & $\approx 1.97(2.00)$ \\
& $k=4$ & $8.63 \mathrm{e}-07$ & $2.24 \mathrm{e}-07$ & $5.77 \mathrm{e}-08$ & $1.47 \mathrm{e}-08$ & $3.74 \mathrm{e}-09$ & $\approx 1.98(2.00)$ \\
& $k=5$ & $1.52 \mathrm{e}-06$ & $3.93 \mathrm{e}-07$ & $1.01 \mathrm{e}-07$ & $2.57 \mathrm{e}-08$ & $6.53 \mathrm{e}-09$ & $\approx 1.98(2.00)$ \\
& $k=6$ & $8.57 \mathrm{e}-09$ & $2.15 \mathrm{e}-09$ & $5.38 \mathrm{e}-10$ & $1.34 \mathrm{e}-10$ & $3.37 \mathrm{e}-11$ & $\approx 2.00(2.00)$ \\
\hline \multirow{5}{*}{0.7} & $k=2$ & $3.13 \mathrm{e}-06$ & $7.88 \mathrm{e}-07$ & $1.98 \mathrm{e}-07$ & $4.97 \mathrm{e}-08$ & $1.25 \mathrm{e}-08$ & $\approx 2.00(2.00)$ \\
& $k=3$ & $8.57 \mathrm{e}-08$ & $1.97 \mathrm{e}-08$ & $4.13 \mathrm{e}-09$ & $8.31 \mathrm{e}-10$ & $1.63 \mathrm{e}-10$ & $\approx 2.35(2.40)$ \\
& $k=4$ & $1.05 \mathrm{e}-07$ & $1.99 \mathrm{e}-08$ & $3.79 \mathrm{e}-09$ & $7.20 \mathrm{e}-10$ & $1.37 \mathrm{e}-10$ & $\approx 2.39(2.40)$ \\
& $k=5$ & $1.55 \mathrm{e}-07$ & $2.97 \mathrm{e}-08$ & $5.66 \mathrm{e}-09$ & $1.08 \mathrm{e}-09$ & $2.05 \mathrm{e}-10$ & $\approx 2.39(2.40)$ \\
& $k=6$ & $1.05 \mathrm{e}-08$ & $2.00 \mathrm{e}-09$ & $3.78 \mathrm{e}-10$ & $7.18 \mathrm{e}-11$ & $1.36 \mathrm{e}-11$ & $\approx 2.40(2.40)$ \\
\hline
\end{tabular}

TABLE 3

Uncorrected BDFk scheme (1.2) at $T=1$ with $h=1 / 100$ and $\tau=1 /\left(100 \times 2^{\ell}\right)$

\begin{tabular}{|c|c|ccccc|c|}
\hline$\alpha$ & $k \backslash \ell$ & 0 & 1 & 2 & 3 & 4 & rate \\
\hline \multirow{5}{*}{0.3} & $k=2$ & $6.01 \mathrm{e}-05$ & $2.99 \mathrm{e}-05$ & $1.49 \mathrm{e}-05$ & $7.47 \mathrm{e}-06$ & $3.73 \mathrm{e}-06$ & $\approx 1.00(1.00)$ \\
& $k=3$ & $5.99 \mathrm{e}-05$ & $2.99 \mathrm{e}-05$ & $1.49 \mathrm{e}-05$ & $7.46 \mathrm{e}-06$ & $3.73 \mathrm{e}-06$ & $\approx 1.00(1.00)$ \\
& $k=4$ & $5.99 \mathrm{e}-05$ & $2.99 \mathrm{e}-05$ & $1.49 \mathrm{e}-05$ & $7.45 \mathrm{e}-06$ & $3.73 \mathrm{e}-06$ & $\approx 1.00(1.00)$ \\
& $k=5$ & $5.98 \mathrm{e}-05$ & $2.99 \mathrm{e}-05$ & $1.49 \mathrm{e}-05$ & $7.45 \mathrm{e}-06$ & $3.72 \mathrm{e}-06$ & $\approx 1.00(1.00)$ \\
& $k=6$ & $9.72 \mathrm{e}-06$ & $4.85 \mathrm{e}-06$ & $2.43 \mathrm{e}-06$ & $1.21 \mathrm{e}-06$ & $6.06 \mathrm{e}-07$ & $\approx 1.00(1.00)$ \\
\hline \multirow{5}{*}{0.5} & $k=2$ & $1.05 \mathrm{e}-04$ & $5.24 \mathrm{e}-05$ & $2.61 \mathrm{e}-05$ & $1.31 \mathrm{e}-05$ & $6.53 \mathrm{e}-06$ & $\approx 1.00(1.00)$ \\
& $k=3$ & $1.05 \mathrm{e}-04$ & $5.23 \mathrm{e}-05$ & $2.61 \mathrm{e}-05$ & $1.31 \mathrm{e}-05$ & $6.53 \mathrm{e}-06$ & $\approx 1.00(1.00)$ \\
& $k=4$ & $1.05 \mathrm{e}-04$ & $5.22 \mathrm{e}-05$ & $2.61 \mathrm{e}-05$ & $1.31 \mathrm{e}-05$ & $6.53 \mathrm{e}-06$ & $\approx 1.00(1.00)$ \\
& $k=5$ & $1.05 \mathrm{e}-04$ & $5.22 \mathrm{e}-05$ & $2.61 \mathrm{e}-05$ & $1.31 \mathrm{e}-05$ & $6.53 \mathrm{e}-06$ & $\approx 1.00(1.00)$ \\
& $k=6$ & $3.85 \mathrm{e}-05$ & $1.92 \mathrm{e}-05$ & $9.62 \mathrm{e}-06$ & $4.81 \mathrm{e}-06$ & $2.40 \mathrm{e}-06$ & $\approx 1.00(1.00)$ \\
\hline \multirow{5}{*}{0.7} & $k=2$ & $1.60 \mathrm{e}-04$ & $8.00 \mathrm{e}-05$ & $3.99 \mathrm{e}-05$ & $2.00 \mathrm{e}-05$ & $9.97 \mathrm{e}-06$ & $\approx 1.00(1.00)$ \\
& $k=3$ & $1.60 \mathrm{e}-04$ & $7.98 \mathrm{e}-05$ & $3.99 \mathrm{e}-05$ & $1.99 \mathrm{e}-05$ & $9.97 \mathrm{e}-06$ & $\approx 1.00(1.00)$ \\
& $k=4$ & $1.60 \mathrm{e}-04$ & $7.98 \mathrm{e}-05$ & $3.99 \mathrm{e}-05$ & $1.99 \mathrm{e}-05$ & $9.97 \mathrm{e}-06$ & $\approx 1.00(1.00)$ \\
& $k=5$ & $1.60 \mathrm{e}-04$ & $7.98 \mathrm{e}-05$ & $3.99 \mathrm{e}-05$ & $1.99 \mathrm{e}-05$ & $9.97 \mathrm{e}-06$ & $\approx 1.00(1.00)$ \\
& $k=6$ & $1.10 \mathrm{e}-04$ & $5.50 \mathrm{e}-05$ & $2.75 \mathrm{e}-05$ & $1.38 \mathrm{e}-05$ & $6.88 \mathrm{e}-06$ & $\approx 1.00(1.00)$ \\
\hline
\end{tabular}

Acknowledgements. The authors are grateful to Prof. Buyang Li for his suggestion and valuable comments on an earlier version of the paper.

\section{REFERENCES}

[1] M. Al-Maskari and S. Karaa. Numerical approximation of semilinear subdiffusion equations with nonsmooth initial data. SIAM J. Numer. Anal., 57(3):1524-1544, 2019. 
[2] A. A. Alikhanov. A new difference scheme for the time fractional diffusion equation. Journal of Computational Physics, 280:424-438, 2015.

[3] N. Y. Bakaev. Maximum norm resolvent estimates for elliptic finite element operators. BIT, 41(2):215-239, 2001.

[4] N. Y. Bakaev, V. Thomée, and L. B. Wahlbin. Maximum-norm estimates for resolvents of elliptic finite element operators. Math. Comp., 72(244):1597-1610, 2003.

[5] B. Berkowitz, J. Klafter, R. Metzler, and H. Scher. Physical pictures of transport in heterogeneous media: Advection-dispersion, random-walk, and fractional derivative formulations. Water Resources Research, 38(10):9-1, 2002.

[6] F. Chen, Q. Xu, and J. S. Hesthaven. A multi-domain spectral method for time-fractional differential equations. J. Comput. Phys., 293:157-172, 2015.

[7] M. Crouzeix and V. Thomée. On the discretization in time of semilinear parabolic equations with nonsmooth initial data. Math. Comp., 49(180):359-377, 1987.

[8] E. Cuesta, C. Lubich, and C. Palencia. Convolution quadrature time discretization of fractional diffusion-wave equations. Math. Comp., 75(254):673-696, 2006.

[9] J. Douglas, Jr., T. Dupont, and L. Wahlbin. The stability in $L^{q}$ of the $L^{2}$-projection into finite element function spaces. Numer. Math., 23:193-197, 1974/75.

[10] Q. Du, J. Yang, and Z. Zhou. An analysis of nonlocal-in-time allen-cahn equations. Preprint.

[11] C. M. Elliott and S. Larsson. Error estimates with smooth and nonsmooth data for a finite element method for the Cahn-Hilliard equation. Math. Comp., 58(198):603-630, S33-S36, 1992.

[12] G. Gao, Z. Sun, and H. Zhang. A new fractional numerical differentiation formula to approximate the Caputo fractional derivative and its applications. J. Comput. Phys., 259:33-50, 2014.

[13] E. Hairer and G. Wanner. Solving ordinary differential equations. II, volume 14 of Springer Series in Computational Mathematics. Springer-Verlag, Berlin, second edition, 1996. Stiff and differential-algebraic problems.

[14] B. Jin, R. Lazarov, and Z. Zhou. Error estimates for a semidiscrete finite element method for fractional order parabolic equations. SIAM J. Numer. Anal., 51(1):445-466, 2013.

[15] B. Jin, R. Lazarov, and Z. Zhou. An analysis of the L1 scheme for the subdiffusion equation with nonsmooth data. IMA J. Numer. Anal., 36(1):197-221, 2016.

[16] B. Jin, R. Lazarov, and Z. Zhou. Two fully discrete schemes for fractional diffusion and diffusion-wave equations with nonsmooth data. SIAM J. Sci. Comput., 38(1):A146-A170, 2016.

[17] B. Jin, R. Lazarov, and Z. Zhou. Numerical methods for time-fractional evolution equations with nonsmooth data: a concise overview. Comput. Methods Appl. Mech. Engrg., 346:332-358, 2019.

[18] B. Jin, B. Li, and Z. Zhou. An analysis of the Crank-Nicolson method for subdiffusion. IMA J. Numer. Anal., 38(1):518-541, 2017.

[19] B. Jin, B. Li, and Z. Zhou. Correction of high-order BDF convolution quadrature for fractional evolution equations. SIAM J. Sci. Comput., 39(6):A3129-A3152, 2017.

[20] B. Jin, B. Li, and Z. Zhou. Numerical analysis of nonlinear subdiffusion equations. SIAM J. Numer. Anal., $56(1): 1-23,2018$.

[21] A. A. Kilbas, H. M. Srivastava, and J. J. Trujillo. Theory and Applications of Fractional Differential Equations. Elsevier Science B.V., Amsterdam, 2006.

[22] S. C. Kou et al. Stochastic modeling in nanoscale biophysics: subdiffusion within proteins. The Annals of Applied Statistics, 2(2):501-535, 2008.

[23] B. Li, K. Wang, and Z. Zhou. Long-time Accurate Symmetrized Implicit-explicit BDF Methods for a Class of Parabolic Equations with Non-self-adjoint Operators. SIAM J. Numer. Anal., 58(1):189-210, 2020.

[24] X. Li and C. Xu. A space-time spectral method for the time fractional diffusion equation. SIAM J. Numer. Anal., 47(3):2108-2131, 2009.

[25] H.-l. Liao, D. Li, and J. Zhang. Sharp error estimate of the nonuniform L1 formula for linear reaction-subdiffusion equations. SIAM J. Numer. Anal., 56(2):1112-1133, 2018.

[26] Y. Lin and C. Xu. Finite difference/spectral approximations for the time-fractional diffusion equation. Journal of Computational Physics, 225(2):1533-1552, 2007.

[27] C. Lubich. Discretized fractional calculus. SIAM Journal on Mathematical Analysis, 17(3):704-719, 1986.

[28] C. Lubich. Convolution quadrature and discretized operational calculus. i. Numerische Mathematik, 52(2):129$145,1988$.

[29] C. Lubich, I. H. Sloan, and V. Thomée. Nonsmooth data error estimates for approximations of an evolution equation with a positive-type memory term. Math. Comp., 65(213):1-17, 1996.

[30] W. McLean and K. Mustapha. Time-stepping error bounds for fractional diffusion problems with non-smooth initial data. J. Comput. Phys., 293:201-217, 2015.

[31] R. Metzler, J.-H. Jeon, A. G. Cherstvy, and E. Barkai. Anomalous diffusion models and their properties: nonstationarity, non-ergodicity, and ageing at the centenary of single particle tracking. Phys. Chem. Chem. Phys., 16:24128, 37 pp., 2014.

[32] K. Mustapha, B. Abdallah, and K. M. Furati. A discontinuous Petrov-Galerkin method for time-fractional diffusion equations. SIAM J. Numer. Anal., 52(5):2512-2529, 2014.

[33] K. Mustapha and W. McLean. Superconvergence of a discontinuous Galerkin method for fractional diffusion and 
wave equations. SIAM J. Numer. Anal., 51(1):491-515, 2013.

[34] R. Nigmatullin. The realization of the generalized transfer equation in a medium with fractal geometry. physica status solidi (b), 133(1):425-430, 1986.

[35] E.-M. Ouhabaz. Gaussian estimates and holomorphy of semigroups. Proc. Amer. Math. Soc., 123(5):1465-1474, 1995.

[36] I. Podlubny. Fractional differential equations: an introduction to fractional derivatives, fractional differential equations, to methods of their solution and some of their applications, volume 198. Elsevier, 1998.

[37] E. Sousa. How to approximate the fractional derivative of order $1<\alpha \leq 2$. International Journal of Bifurcation and Chaos, 22(04):1250075, 2012.

[38] H. B. Stewart. Generation of analytic semigroups by strongly elliptic operators. Trans. Amer. Math. Soc., 199:141-162, 1974.

[39] M. Stynes, E. O'Riordan, and J. L. Gracia. Error analysis of a finite difference method on graded meshes for a time-fractional diffusion equation. SIAM J. Numer. Anal., 55(2):1057-1079, 2017.

[40] Z. Sun and X. Wu. A fully discrete difference scheme for a diffusion-wave system. Applied Numerical Mathematics, 56(2):193-209, 2006.

[41] V. Thomée. Galerkin finite element methods for parabolic problems (springer series in computational mathematics). 2006.

[42] R. Wu, H. Ding, and C. Li. Determination of coefficients of high-order schemes for riemann-liouville derivative. The Scientific World Journal, 2014, 2014.

[43] Y. Yan, M. Khan, and N. J. Ford. An analysis of the modified L1 scheme for time-fractional partial differential equations with nonsmooth data. SIAM J. Numer. Anal., 56(1):210-227, 2018.

[44] S. B. Yuste and L. Acedo. An explicit finite difference method and a new von Neumann-type stability analysis for fractional diffusion equations. SIAM J. Numer. Anal., 42(5):1862-1874, 2005.

[45] M. Zayernouri, M. Ainsworth, and G. E. Karniadakis. A unified Petrov-Galerkin spectral method for fractional PDEs. Comput. Methods Appl. Mech. Engrg., 283:1545-1569, 2015.

[46] F. Zeng, C. Li, F. Liu, and I. Turner. The use of finite difference/element approaches for solving the time-fractional subdiffusion equation. SIAM J. Sci. Comput., 35(6):A2976-A3000, 2013. 